\title{
An efficient one-pot synthesis of carbazole fused benzoquinolines and pyridocarbazoles
}

\author{
KRIPALAYA RATHEESH ARYA ${ }^{\mathrm{a}}$, HAZEL A SPARKES ${ }^{\mathrm{b}}$ and \\ KARNAM JAYARAM PILLAI RAJENDRA PRASAD ${ }^{\mathrm{a}, *}$ \\ ${ }^{a}$ Department of Chemistry, Bharathiar University, Coimbatore, Tamilnadu 641 046, India \\ ${ }^{\mathrm{b}}$ School of Chemistry, University of Bristol, Cantock's Close, Bristol BS8 1TS, UK \\ E-mail: prasad_125@yahoo.com
}

MS received 1 November 2017; revised 3 January 2018; accepted 23 January 2018; published online 28 March 2018

\begin{abstract}
A one-pot, solvent-free protocol for the synthesis of chloro-substituted benzoquinoline-carbazole derivatives via a modified Friedländer hetero-annulation reaction between 2, 3, 4, 9-tetrahydrocarbazol-1-one and 3-amino-2-naphthoic acid in the presence of $\mathrm{POCl}_{3}$ is described. In addition, the direct pseudo multicomponent transformation of 2, 3, 4, 9-tetrahydrocarbazol-1-one, malononitrile and 9-ethyl-3-carbazolecarboxaldehyde results in the formation of a multifunctionalized carbazole through a Knoevenagel-Michael addition-cyclization reaction has also been reported. All the newly synthesized molecules were deduced by spectral and analytical methods.
\end{abstract}

Keywords. Modified Friedländer reaction; benzoquinoline-carbazoles; pseudo multicomponent reaction; carbazole substituted pyridocarbazole.

\section{Introduction}

The structural diversity and biological importance of nitrogen containing heterocycles such as carbazole and quinoline derivatives have made them attractive targets in both medicinal and organic chemistry. The heteroarylcarbazole derivatives have been found to display a diverse array of important functions and are abundant in bioactive natural products. ${ }^{1-4}$ For example, the pyridocarbazole type alkaloids, ellipticine(extracted from the leaves of Ochrosia elliptica) and Olivacine (isolated from Aspidosperma olivaceum) exhibit a wide spectrum of biological and medicinal activities. ${ }^{5-9}$ The benzoquinoline core structure is also found in a wide variety of biologically active natural products and pharmaceuticals with anti-Parkinson, antipsychotic, antibacterial, UDP (Uridine diphosphate)-glucuronosyl transferase, antimalarial, agonistic and antipsychotic activities. ${ }^{10-14}$ Kantevari et al., reported that the coupling of 9methyl-9H-carbazole to tetrahydroquinoline nucleus could deliver a new scaffold with better antimycobacterial activity than its individual reactants. ${ }^{15}$ Recently our research group reported carbazole and quinoline-based hybrid moieties for its pharmacological interest. ${ }^{16}$

As an extension to our efforts in developing heterocycles of biological interest and also considering the significant role of carbazoles and benzoquinolines in biological applications, we were inspired to synthesize a new series of benzoquinoline-carbazole and carbazole dimer from 2,3,4,9-tetrahydro- $1 H$-carbazol1 -one as the precursor via a one-pot protocol.

\section{Experimental}

\subsection{General}

All the chemicals were bought from Sigma-Aldrich and Merck and were utilized for the process without further purification. Melting points (M.p.) were determined on a Mettler FP 51 apparatus (Mettler Instruments, Switzerland) and are uncorrected. They are expressed in degree centigrade $\left({ }^{\circ} \mathrm{C}\right)$. FT-IR spectra were recorded on Avatar Model FT-IR (4000-400 $\mathrm{cm}^{-1}$ ) spectrophotometer. ${ }^{1} \mathrm{H}$ NMR and ${ }^{13} \mathrm{C}$ NMR spectra were recorded on an Agilent- $400 \mathrm{MHz}$

\footnotetext{
*For correspondence

Electronic supplementary material: The online version of this article (https://doi.org/10.1007/s12039-018-1428-1) contains supplementary material, which is available to authorized users.
} 
$\left({ }^{1} \mathrm{H}\right)$ and $100 \mathrm{MHz}\left({ }^{13} \mathrm{C}\right)$ spectrometers respectively in $\mathrm{CDCl}_{3}$ using TMS (tetramethylsilane) as internal reference; chemical shifts are expressed in parts per million (ppm); coupling constants $(J)$ are reported in hertz $(\mathrm{Hz})$ and the terms $J_{o}$ and $J_{m}$ refer to ortho coupling constant and meta coupling constant. The signals were characterized as $\mathrm{s}$ (singlet), $\mathrm{d}$ (doublet), $\mathrm{t}$ (triplet), m (multiplet), bs (broad singlet) and dd (doublet of doublet). Microanalyses were carried out using Vario EL III model CHNS analyzer (Vario, Germany). Absorption spectral measurements were carried out using JASCO V-630 UVVisible spectrophotometer. Quartz cuvettes of path length $1 \mathrm{~cm}$ were used to record the absorption spectra. The emission spectral studies were performed with JASCO FP-6600 spectrofluorometer equipped with a $1 \mathrm{~cm}$ quartz cuvette at the Department of Chemistry, Bharathiar University. When known compounds had to be prepared according to literature procedures pertinent references are given. The purity of the products was tested by TLC plates coated with silica gel-G using petroleum ether and ethyl acetate in the ratio of $1: 1$ as developing solvents.

\subsection{General procedure for the synthesis of 7-chloro-} benzo[6 $\left.6^{\prime}, 7^{\prime}-a^{\prime}\right]$ quino $\left[2^{\prime}, 3^{\prime}-a\right]-5,6$-dihydrocarbazole 3

A mixture of 2,3,4,9-tetrahydrocarbazol-1-one 1 (1.0 mmol) and 3-amino-2-naphthoic acid $2(1.0 \mathrm{mmol})$ was refluxed with phosphorus oxychloride $(5 \mathrm{~mL})$ for $5 \mathrm{~h}$ at $120^{\circ} \mathrm{C}$. The completeness of the reaction was monitored by TLC. After completion, the reaction mixture was poured into ice water and extracted with ethyl acetate. Combined organic layers were dried over anhydrous magnesium sulphate. It was then purified on a silica-gel column chromatography (eluent: petroleum ether/ethyl acetate, 99:1).

\subsection{The spectral and analytical data of all the compounds $\mathbf{3}(\boldsymbol{a}-\boldsymbol{d})$}

2.3a 7-Chloro-benzo $\left[6^{\prime}, 7^{\prime}-a^{\prime}\right]$ quino $\left[2^{\prime}, 3^{\prime}\right.$-a]-5,6-dihy drocarbazole (3a): White solid; yield: $283 \mathrm{mg}(80 \%)$; M.p. $201-203{ }^{\circ} \mathrm{C}$; FT-IR $\left(\mathrm{KBr}, \mathrm{cm}^{-1}\right) v_{\max }: 3436(\mathrm{NH})$, $1592(\mathrm{C}=\mathrm{N}) ;{ }^{1} \mathrm{H}$ NMR $\left(400 \mathrm{MHz}, \mathrm{CDCl}_{3}\right)(\mathrm{ppm}) \delta_{\mathrm{H}}: 9.47$ (br s, 1H, NH), 8.66 (s, 1H, ArH), 8.52 (s, 1H, ArH), 8.04-7.98 $(\mathrm{m}, 2 \mathrm{H}, \mathrm{ArH}), 7.62\left(\mathrm{~d}, 1 \mathrm{H}, \mathrm{ArH}, J_{o}=7.60 \mathrm{~Hz}\right), 7.52-7.47$ $(\mathrm{m}, 2 \mathrm{H}, \mathrm{ArH}), 7.39$ (d, 1H, ArH, $\left.J_{o}=8.40 \mathrm{~Hz}\right), 7.29-7.27$ $(\mathrm{m}, 1 \mathrm{H}, \mathrm{ArH}), 7.13\left(\mathrm{t}, 1 \mathrm{H}, \mathrm{ArH}, J_{o}=7.20 \mathrm{~Hz}\right), 3.51-$ $3.48\left(\mathrm{~m}, 2 \mathrm{H}, \mathrm{CH}_{2}\right), 3.21-3.17\left(\mathrm{~m}, 2 \mathrm{H}, \mathrm{CH}_{2}\right) ;{ }^{13} \mathrm{C} \mathrm{NMR}(100$ $\left.\mathrm{MHz}, \mathrm{CDCl}_{3}\right)(\mathrm{ppm}) \delta_{\mathrm{C}}: 149.2,148.4,143.8,138.6,138.1$, 134.0, 132.4, 131.6, 128.5, 127.9, 126.8, 126.6, 126.0, 124.7, 124.5, 123.5, 120.0, 119.8, 111.8, 26.7, 19.0; Anal. calcd. for $\mathrm{C}_{23} \mathrm{H}_{15} \mathrm{ClN}_{2}$ : C, 77.85; H, 4.26; N, 7.89; found: C, 77.94; H, $4.22 ; \mathrm{N}, 7.83 \%$.

$2.3 \mathrm{~b}$ 7-Chloro-3-methyl-benzo[6 $\left.6^{\prime}, 7^{\prime}-a^{\prime}\right]$ quino $\left[2^{\prime}, 3^{\prime}-a\right]$ -5,6-dihydrocarbazole ( $3 \boldsymbol{b}$ ): White solid; yield: $253 \mathrm{mg}$ (69\%); M.p. $198-200{ }^{\circ} \mathrm{C}$; FT-IR $\left(\mathrm{KBr}, \mathrm{cm}^{-1}\right) v_{\max }: 3462$ $(\mathrm{NH}), 1592(\mathrm{C}=\mathrm{N}) ;{ }^{1} \mathrm{H}$ NMR $\left(400 \mathrm{MHz}, \mathrm{CDCl}_{3}\right)(\mathrm{ppm}) \delta_{\mathrm{H}}$ : 9.34 (br s, 1H, NH), 8.64 (s, 1H, ArH), 8.49 (s, 1H, ArH),
8.03-7.97 (m, 2H, ArH), 7.51-7.46 (m, 2H, ArH), 7.38 (s, 1H, $\mathrm{ArH}), 7.25\left(\mathrm{~d}, 1 \mathrm{H}, \mathrm{ArH}, J_{o}=8.80 \mathrm{~Hz}\right), 7.08(\mathrm{~d}, 1 \mathrm{H}, \mathrm{ArH}$, $\left.J_{o}=8.80 \mathrm{~Hz}\right), 3.49-3.45\left(\mathrm{~m}, 2 \mathrm{H}, \mathrm{CH}_{2}\right), 3.17-3.13(\mathrm{~m}, 2 \mathrm{H}$, $\left.\mathrm{CH}_{2}\right), 2.45\left(\mathrm{~s}, 3 \mathrm{H}, \mathrm{CH}_{3}\right) ;{ }^{13} \mathrm{C} \mathrm{NMR}\left(100 \mathrm{MHz}, \mathrm{CDCl}_{3}\right)(\mathrm{ppm})$ $\delta_{\mathrm{C}}: 148.5,143.9,138.4,136.5,134.0,132.7,131.6,129.3$, $128.5,127.9,127.0,126.5,126.1,125.9,124.5,123.5,119.3$, 111.4, 26.7, 21.4, $19.0\left(\mathrm{CH}_{3}\right)$; Anal. calcd. for $\mathrm{C}_{24} \mathrm{H}_{17} \mathrm{ClN}_{2}$ : C, 78.15; H, 4.65; N, 7.59; Found: C, 78.24; H, 4.63; N, $7.65 \%$.

$2.3 \mathrm{c}$ 7-Chloro-1-methyl-benzo[6 $\left.6^{\prime}, 7^{\prime}-a^{\prime}\right]$ quino $\left[2^{\prime}, 3^{\prime}-a\right]$ -5,6-dihydrocarbazole (3c): White solid; yield: $246 \mathrm{mg}$ (67\%); M.p. $199-201{ }^{\circ} \mathrm{C}$; FT-IR $\left(\mathrm{KBr}, \mathrm{cm}^{-1}\right) v_{\max }: 3281$ $(\mathrm{NH}), 1590(\mathrm{C}=\mathrm{N}) ;{ }^{1} \mathrm{H}$ NMR $\left(400 \mathrm{MHz}, \mathrm{CDCl}_{3}\right)(\mathrm{ppm}) \delta_{\mathrm{H}}$ : 9.13 (br s, 1H, NH), 8.68 (s, 1H, ArH), 8.55 (s, 1H, ArH), 8.05-8.12 (m, 2H, ArH), 7.54-7.47 (m, 3H, ArH), 7.12-7.05 (m, 2H, ArH), 3.52-3.49 (m, 2H, $\left.\mathrm{CH}_{2}\right), 3.22-3.19$ (m, 2H, $\mathrm{CH}_{2}$ ), 2.59 (s, $\left.3 \mathrm{H}, \mathrm{CH}_{3}\right)$; Anal. calcd. for $\mathrm{C}_{24} \mathrm{H}_{17} \mathrm{ClN}_{2}$ : $\mathrm{C}$, 78.15; H, 4.65; N, 7.59; Found: C, 78.22; H, 4.69; N, 7.63\%.

2.3d 3,7-Dichloro-benzo [6', 7'- $\left.a^{\prime}\right]$ quino $\left[2^{\prime}, 3^{\prime}-a\right]-5,6$ -dihydrocarbazole (3d): White solid; yield: $213 \mathrm{mg}$ (55\%); M.p. $195-197{ }^{\circ} \mathrm{C}$; FT-IR $\left(\mathrm{KBr}, \mathrm{cm}^{-1}\right) v_{\max }: 3263$ $(\mathrm{NH}), 1549(\mathrm{C}=\mathrm{N}) ;{ }^{1} \mathrm{H}$ NMR $\left(400 \mathrm{MHz}, \mathrm{CDCl}_{3}\right)(\mathrm{ppm}) \delta_{\mathrm{H}}$ : 8.69 (br s, $1 \mathrm{H}, \mathrm{NH}), 8.60$ (s, 1H, ArH), 8.06-8.01 (m, 2H, $\mathrm{ArH}), 7.58(\mathrm{~m}, 1 \mathrm{H}, \mathrm{ArH}), 7.55-7.53(\mathrm{~m}, 2 \mathrm{H}, \mathrm{ArH}), 7.39$ $\left(\mathrm{d}, 2 \mathrm{H}, \mathrm{ArH}, J_{o}=8.40 \mathrm{~Hz}\right), 7.25(\mathrm{~s}, 1 \mathrm{H}, \mathrm{ArH}), 3.53-3.49$ (m, 2H, $\left.\mathrm{CH}_{2}\right), 3.19-3.15\left(\mathrm{~m}, 2 \mathrm{H}, \mathrm{CH}_{2}\right)$, Anal. calcd. for $\mathrm{C}_{23} \mathrm{H}_{14} \mathrm{Cl}_{2} \mathrm{~N}_{2}$ : C, 70.96; H, 3.62; N, 7.20; Found: C, 70.87; $\mathrm{H}, 3.66 ; \mathrm{N}, 7.27 \%$.

\subsection{General procedure for the preparation of carbazole substituted pyrido[2,3-a]carbazoles 6 and 7}

A mixture of 2,3,4,9-tetrahydrocarbazol-1-one $\mathbf{1}(1.0 \mathrm{mmol})$, malononitrile 4, (1.0 mmol), 9-ethyl-3-carbazole carboxaldehyde 5 (1.0 mmol) and lithium ethoxide (3 equiv.) in $15 \mathrm{~mL}$ of ethanol $\backslash$ methanol was heated to reflux for $3 \mathrm{~h}$. The reaction was monitored by TLC which indicated the formation of the product. The excess of solvent was removed by distillation and the mixture was poured into ice-water. The reaction mixture was then neutralized with $5 \mathrm{~N} \mathrm{HCl}$ and extracted with ethyl acetate. The organic layer was thoroughly washed with water and dried over anhydrous $\mathrm{Na}_{2} \mathrm{SO}_{4}$. Upon removal of the solvent, a brown crude mixture was obtained. It was purified by column chromatography over silica gel using petroleum ether: ethyl acetate (97:3) mixture as eluent to afford the corresponding product, 2-ethoxy/methoxy-4-aryl/heteroaryl-5,6dihydro-11 $H$-pyrido[2,3- $a$ ]carbazole-3-carbonitrile 6 \& 7.

\subsection{The spectral and analytical data of all the compounds 6 \& 7}

2.5a 2-Ethoxy-4-(9'-ethyl-9H-carbazol-3'-yl)-5,6-dih ydro-11H-pyrido[2,3-a]carbazole-3-carbonitrile (6a): Yellow solid; yield: $351 \mathrm{mg}$ (73\%); M.p. $272-274{ }^{\circ} \mathrm{C}$; FTIR $\left(\mathrm{KBr}, \mathrm{cm}^{-1}\right) \nu_{\max }$ : $3259(\mathrm{NH}), 2216(\mathrm{CN}), 1544(\mathrm{C}=\mathrm{N})$; 
${ }^{1} \mathrm{H}$ NMR $\left(400 \mathrm{MHz} \mathrm{CDCl}_{3}\right)(\mathrm{ppm}) \delta_{\mathrm{H}}: 8.81$ (br s, $\left.1 \mathrm{H}, \mathrm{NH}\right)$, $8.10(\mathrm{~d}, 1 \mathrm{H}, \mathrm{ArH}, J=8.00 \mathrm{~Hz}), 8.06$ (s, 1H, ArH), 7.587.44 (m, 6H, ArH), 7.29-7.27 (m, 1H, ArH), 7.15-7.11 (m, $2 \mathrm{H}, \mathrm{ArH}), 4.64$ (q, 2H, $\left.\mathrm{C}_{2}-\mathrm{OCH}_{2} \mathrm{CH}_{3}, J=7.20 \mathrm{~Hz}\right), 4.42$ $\left(\mathrm{q}, 2 \mathrm{H}, \mathrm{N}_{9}{ }^{\prime}-\mathrm{CH}_{2} \mathrm{CH}_{3}, J=7.20 \mathrm{~Hz}\right), 2.97-2.94(\mathrm{~m}, 4 \mathrm{H}$, $\left.\mathrm{CH}_{2}\right), 1.54-1.48\left(\mathrm{~m}, 6 \mathrm{H}, \mathrm{C}_{2}-\mathrm{OCH}_{2} \mathrm{CH}_{3} \& \mathrm{~N}_{9}{ }^{\prime}-\mathrm{CH}_{2} \mathrm{CH}_{3}\right)$; ${ }^{13} \mathrm{C} \mathrm{NMR}\left(100 \mathrm{MHz}, \mathrm{CDCl}_{3}\right)(\mathrm{ppm}) \delta_{\mathrm{C}}: 163.5,155.4,148.4$, 140.4, 140.0, 137.8, 132.4, 126.8, 126.1, 126.0, 125.8, 124.4, $122.9,122.7,121.9,120.7,120.6,120.2,119.8,119.2,118.6$ $(\mathrm{CN}), 116.1,111.7,108.7,108.6,93.9,63.1\left(\mathrm{C}_{2}-\mathrm{OCH}_{2} \mathrm{CH}_{3}\right)$, $37.7\left(\mathrm{~N}_{9}{ }^{-}-\mathrm{CH}_{2} \mathrm{CH}_{3}\right), 25.4,19.4,14.6\left(\mathrm{C}_{2}-\mathrm{OCH}_{2} \mathrm{CH}_{3}\right), 13.8$ ( $\mathrm{N}_{9}{ }^{\prime}-\mathrm{CH}_{2} \mathrm{CH}_{3}$ ); Anal. calcd. for $\mathrm{C}_{32} \mathrm{H}_{26} \mathrm{~N}_{4} \mathrm{O}: \mathrm{C}, 79.64 ; \mathrm{H}$, 5.43; N, 11.61; Found: C, 79.74; H, 5.47; N, $11.57 \%$.

$2.5 b \quad 2$-Ethoxy-4-(9'-ethyl-9H-carbazol-3'-yl)-10-met hyl-5,6-dihydro-11H-pyrido[2,3-a]carbazole-3-carbo nitrile (6b): Yellow solid; yield: $347 \mathrm{mg}$ (70\%); M.p. 271-273 ${ }^{\circ} \mathrm{C}$; FT-IR $\left(\mathrm{KBr}, \mathrm{cm}^{-1}\right) v_{\max }: 3449(\mathrm{NH}), 2217$ $(\mathrm{CN}), 1551(\mathrm{C}=\mathrm{N}) ;{ }^{1} \mathrm{H}$ NMR $\left(400 \mathrm{MHz}, \mathrm{CDCl}_{3}\right)(\mathrm{ppm}) \delta_{\mathrm{H}}$ : 8.62 (br s, $1 \mathrm{H}, \mathrm{NH}), 8.10$ (d, 1H, ArH, $J=8.00 \mathrm{~Hz}$ ), 8.06 (s, 1H, ArH), 7.54-7.41 (m, 5H, ArH), 7.26-7.25 (m, 1H, $\mathrm{ArH}), 7.07(\mathrm{~d}, 2 \mathrm{H}, \mathrm{ArH}, J=8.00 \mathrm{~Hz}), 4.66\left(\mathrm{q}, 2 \mathrm{H}, \mathrm{C}_{2}-\right.$ $\left.\mathrm{OCH}_{2} \mathrm{CH}_{3}, J=7.20 \mathrm{~Hz}\right), 4.42\left(\mathrm{q}, 2 \mathrm{H}, \mathrm{N}_{9}{ }^{\prime}-\mathrm{CH}_{2} \mathrm{CH}_{3}, J=\right.$ $6.80 \mathrm{~Hz}), 2.94-2.93\left(\mathrm{~m}, 4 \mathrm{H}, \mathrm{CH}_{2}\right), 2.59\left(\mathrm{~s}, 3 \mathrm{H}, \mathrm{CH}_{3}\right), 1.54$ $1.47\left(\mathrm{~m}, 6 \mathrm{H}, \mathrm{C}_{2}-\mathrm{OCH}_{2} \mathrm{CH}_{3} \& \mathrm{~N}_{9}{ }^{\prime}-\mathrm{CH}_{2} \mathrm{CH}_{3}\right) ;{ }^{13} \mathrm{C} \mathrm{NMR}(100$ $\left.\mathrm{MHz}, \mathrm{CDCl}_{3}\right)(\mathrm{ppm}) \delta_{\mathrm{C}}: 163.5,155.4,148.5,140.4,140.0$, $137.4,132.1,126.1,126.1,126.0,125.9,125.0,122.9,122.7$, $121.9,120.8,120.7,120.6,120.4,119.3,119.2,117.5(\mathrm{CN})$, 116.2, 108.7, 108.6, 93.9, $62.9\left(\mathrm{C}_{2}-\mathrm{OCH}_{2} \mathrm{CH}_{3}\right), 37.7\left(\mathrm{~N}_{9}\right)-$ $\left.\mathrm{CH}_{2} \mathrm{CH}_{3}\right), 25.5,19.5,16.7\left(\mathrm{CH}_{3}\right), 14.6\left(\mathrm{C}_{2}-\mathrm{OCH}_{2} \mathbf{C H}_{3}\right), 13.8$ $\left(\mathrm{N}_{9}\right.$ '- $\mathrm{CH}_{2} \mathrm{CH}_{3}$ ); Anal. calcd. for $\mathrm{C}_{33} \mathrm{H}_{28} \mathrm{~N}_{4} \mathrm{O}: \mathrm{C}, 79.81 ; \mathrm{H}$, 5.68; N, 11.28; Found: C, 79.90; H, 5.64; N, 11.21\%.

2.5c 2-Ethoxy-4-phenyl-5,6-dihydro-11H-pyrido[2,3a]carbazole-3-carbonitrile (6c): Yellow solid; yield: 292 mg (80\%); M.p. $261-263{ }^{\circ} \mathrm{C}$; FT-IR $\left(\mathrm{KBr}, \mathrm{cm}^{-1}\right) v_{\max }$ : $3331(\mathrm{NH}), 2216(\mathrm{CN}), 1552(\mathrm{C}=\mathrm{N}) ;{ }^{1} \mathrm{H}$ NMR $(400 \mathrm{MHz}$, $\left.\mathrm{CDCl}_{3}\right)(\mathrm{ppm}) \delta_{\mathrm{H}}: 8.80($ br s, $1 \mathrm{H}, \mathrm{NH}), 7.57(\mathrm{~d}, 1 \mathrm{H}, \mathrm{ArH}$, $\left.J_{o}=8.00 \mathrm{~Hz}\right), 7.53-7.47(\mathrm{~m}, 3 \mathrm{H}, \mathrm{ArH}), 7.44(\mathrm{~d}, 1 \mathrm{H}, \mathrm{ArH}$, $\left.J_{o}=8.00 \mathrm{~Hz}\right), 7.34\left(\mathrm{~d} \mathrm{~d}, 2 \mathrm{H}, \mathrm{ArH}, J_{m}=1.80 \mathrm{~Hz} \& J_{o}=\right.$ $7.80 \mathrm{~Hz}), 7.28(\mathrm{t}, 1 \mathrm{H}, \mathrm{ArH}, J=7.40 \mathrm{~Hz}), 7.14(\mathrm{t}, 1 \mathrm{H}, \mathrm{ArH}$, $J=7.40 \mathrm{~Hz}), 4.62\left(\mathrm{q}, 2 \mathrm{H}, \mathrm{C}_{2}-\mathrm{OCH}_{2} \mathrm{CH}_{3}, J=7.20 \mathrm{~Hz}\right)$, 2.96-2.92 (m, 2H, $\left.\mathrm{CH}_{2}\right), 2.87-2.83\left(\mathrm{~m}, 2 \mathrm{H}, \mathrm{CH}_{2}\right), 1.51(\mathrm{t}, 3 \mathrm{H}$, $\left.\mathrm{C}_{2}-\mathrm{OCH}_{2} \mathrm{CH}_{3}, J=7.20 \mathrm{~Hz}\right) ;{ }^{13} \mathrm{C} \mathrm{NMR}\left(100 \mathrm{MHz}, \mathrm{CDCl}_{3}\right)$ $(\mathrm{ppm}) \delta_{\mathrm{C}}: 163.4,154.2,148.6,137.8,135.5,132.2,129.2$, 129.0, 128.7, 128.4, 126.9, 126.7, 124.6, 121.3, 120.2, 119.9, $118.8(\mathrm{CN}), 115.7,111.7,93.2,63.0\left(\mathrm{OCH}_{2} \mathrm{CH}_{3}\right), 25.2,19.4$, $14.5\left(\mathrm{OCH}_{2} \mathrm{CH}_{3}\right)$; Anal. calcd. for $\mathrm{C}_{24} \mathrm{H}_{19} \mathrm{~N}_{3} \mathrm{O}: \mathrm{C}, 78.88 ; \mathrm{H}$, 5.24; N, 11.50; Found: C, 78.79; H, 5.28; N, 11.57\%.

\section{5d 2-Ethoxy-10-methyl-4-phenyl-5,6-dihydro- $11 \mathrm{H}$} -pyrido[2,3-a]carbazole-3-carbonitrile (6d): Yellow solid; yield: $299 \mathrm{mg}$ (79\%); M.p. $259-261{ }^{\circ} \mathrm{C}$; FT-IR (KBr, $\left.\mathrm{cm}^{-1}\right) v_{\max }: 3345(\mathrm{NH}), 2218(\mathrm{CN}), 1553(\mathrm{C}=\mathrm{N}) ;{ }^{1} \mathrm{H}$ NMR $\left(400 \mathrm{MHz}, \mathrm{CDCl}_{3}\right)(\mathrm{ppm}) \delta_{\mathrm{H}}: 8.59(\mathrm{br} \mathrm{s}, 1 \mathrm{H}, \mathrm{NH}), 7.54-7.47$ $(\mathrm{m}, 3 \mathrm{H}, \mathrm{ArH}), 7.42\left(\mathrm{~d}, 1 \mathrm{H}, \mathrm{ArH}, J_{o}=6.80 \mathrm{~Hz}\right), 7.35(\mathrm{~d} \mathrm{~d}$, $\left.2 \mathrm{H}, \mathrm{ArH}, J_{m}=1.60 \mathrm{~Hz} \& J_{o}=6.40 \mathrm{~Hz}\right), 7.10-7.04(\mathrm{~m}, 2 \mathrm{H}$,
$\mathrm{ArH}), 4.64$ (q, 2H, $\mathrm{C}_{2}-\mathrm{OCH}_{2} \mathrm{CH}_{3}, J=7.20 \mathrm{~Hz}$ ), 2.95-2.90 $\left(\mathrm{m}, 2 \mathrm{H}, \mathrm{CH}_{2}\right), 2.87-2.83\left(\mathrm{~m}, 2 \mathrm{H}, \mathrm{CH}_{2}\right), 2.58\left(\mathrm{~s}, 3 \mathrm{H}, \mathrm{CH}_{3}\right)$, $1.52\left(\mathrm{t}, 3 \mathrm{H}, \mathrm{C}_{2}-\mathrm{OCH}_{2} \mathrm{CH}_{3}, J=7.20 \mathrm{~Hz}\right)$; Anal. calcd. for $\mathrm{C}_{25} \mathrm{H}_{21} \mathrm{~N}_{3} \mathrm{O}$ : C, 79.13; H, 5.58; N, 11.07; Found: C, 79.20; $\mathrm{H}, 5.54 ; \mathrm{N}, 11.01 \%$.

2.5e 2-Ethoxy-4-(thiophen-2-yl)-5,6-dihydro-11H-py rido[2,3-a]carbazole-3-carbonitrile (6e): Yellow solid; yield: $255 \mathrm{mg}$ (69\%); M.p. $255-257{ }^{\circ} \mathrm{C}$; FT-IR (KBr, $\left.\mathrm{cm}^{-1}\right) \nu_{\max }$ : $3316(\mathrm{NH}), 2216(\mathrm{CN}), 1557(\mathrm{C}=\mathrm{N}) ;{ }^{1} \mathrm{H}$ NMR $\left(400 \mathrm{MHz}, \mathrm{CDCl}_{3}\right)(\mathrm{ppm}) \delta_{\mathrm{H}}: 8.77(\mathrm{br} \mathrm{s}, 1 \mathrm{H}, \mathrm{NH}), 7.58(\mathrm{~d}$, $\left.1 \mathrm{H}, \mathrm{ArH}, J_{o}=8.00 \mathrm{~Hz}\right), 7.53\left(\mathrm{~d} \mathrm{~d}, 1 \mathrm{H}, \mathrm{ArH}, J_{m}=1.20 \mathrm{~Hz}\right.$ $\left.\& J_{o}=4.80 \mathrm{~Hz}\right), 7.43\left(\mathrm{~d}, 1 \mathrm{H}, \mathrm{ArH}, J_{o}=8.00 \mathrm{~Hz}\right), 7.29$ $\left(\mathrm{d} \mathrm{d}, 1 \mathrm{H}, \mathrm{ArH}, J_{m}=1.20 \mathrm{~Hz} \& J_{o}=6.80 \mathrm{~Hz}\right), 7.21-7.12$ (m, 3H, ArH), 4.61 (q, 2H, $\mathrm{C}_{2}-\mathrm{OCH}_{2} \mathrm{CH}_{3}, J=7.20 \mathrm{~Hz}$ ), 3.03-2.96 (m, 4H, $\left.\mathrm{CH}_{2}\right), 1.50$ (t, 3H, $\mathrm{C}_{2}-\mathrm{OCH}_{2} \mathrm{CH}_{3}, J=$ $7.20 \mathrm{~Hz}) ;{ }^{13} \mathrm{C} \mathrm{NMR}\left(100 \mathrm{MHz}, \mathrm{CDCl}_{3}\right)(\mathrm{ppm}) \delta_{\mathrm{C}}: 163.5$, 148.6, 147.0, 137.9, 134.9, 132.1, 129.0, 127.7, 127.4, 126.7, 124.7, 122.6, 120.3, 119.9, $119.1(\mathrm{CN}), 115.5,111.7,94.0$, $63.1\left(\mathrm{OCH}_{2} \mathrm{CH}_{3}\right), 25.4,19.3,14.5\left(\mathrm{OCH}_{2} \mathrm{CH}_{3}\right)$; Anal. calcd. for $\mathrm{C}_{22} \mathrm{H}_{17} \mathrm{~N}_{3} \mathrm{OS}$ : C, 71.14; H, 4.61; N, 11.31; Found: C, 71.23; H, 4.57; N, $11.36 \%$.

\section{5f 2-Ethoxy-10-methyl-4-(thiophen-2-yl)-5,6-dihy} dro-11H-pyrido[2,3-a]carbazole-3-carbonitrile (6f): Yellow solid; yield: $250 \mathrm{mg}(65 \%)$; M.p. $253-255^{\circ} \mathrm{C}$; FTIR $\left(\mathrm{KBr}, \mathrm{cm}^{-1}\right) v_{\max }$ : $3349(\mathrm{NH}), 2214(\mathrm{CN}), 1552(\mathrm{C}=\mathrm{N})$; ${ }^{1} \mathrm{H} \mathrm{NMR}\left(400 \mathrm{MHz}, \mathrm{CDCl}_{3}\right)(\mathrm{ppm}) \delta_{\mathrm{H}}: 8.56(\mathrm{br} \mathrm{s}, 1 \mathrm{H}, \mathrm{NH})$, $7.53\left(\mathrm{~d} \mathrm{~d}, 1 \mathrm{H}, \mathrm{ArH}, J_{m}=1.20 \mathrm{~Hz} \& J_{o}=4.80 \mathrm{~Hz}\right), 7.43(\mathrm{~d}$, $\left.1 \mathrm{H}, \mathrm{ArH}, J_{o}=7.60 \mathrm{~Hz}\right), 7.21-7.14(\mathrm{~m}, 2 \mathrm{H}, \mathrm{ArH}), 7.08-7.04$ $(\mathrm{m}, 2 \mathrm{H}, \mathrm{ArH}), 4.63\left(\mathrm{q}, 2 \mathrm{H}, \mathrm{C}_{2}-\mathrm{OCH}_{2} \mathrm{CH}_{3}, J=7.20 \mathrm{~Hz}\right)$, 3.03-2.95 (m, 4H, $\left.\mathrm{CH}_{2}\right), 2.57$ (s, 3H, $\left.\mathrm{CH}_{3}\right), 1.50$ (t, 3H, $\mathrm{C}_{2}-$ $\mathrm{OCH}_{2} \mathrm{CH}_{3}, J=7.20 \mathrm{~Hz}$ ); Anal. calcd. for $\mathrm{C}_{23} \mathrm{H}_{19} \mathrm{~N}_{3} \mathrm{OS}$ : C, 71.66; H, 4.97; N, 10.90; Found: C, 71.72; H, 4.93; N, $10.97 \%$.

\section{$2.5 \mathrm{~g}$ 2-ethoxy-4-(4'-chlorophenyl)-5,6-dihydro- $11 \mathrm{H}$ -} pyrido[2,3-a]carbazole-3-carbonitrile $(\mathbf{6 g})$ : Yellow solid; yield: $231 \mathrm{mg}(58 \%)$; M.p. $249-250{ }^{\circ} \mathrm{C}$; FT-IR (KBr, $\left.\mathrm{cm}^{-1}\right) \nu_{\max }: 3286(\mathrm{NH}), 2220(\mathrm{CN}), 1644(\mathrm{C}=\mathrm{N}) ;{ }^{1} \mathrm{H}$ NMR $\left(400 \mathrm{MHz}, \mathrm{CDCl}_{3}\right)(\mathrm{ppm}) \delta_{\mathrm{H}}: 8.79(\mathrm{br} \mathrm{s}, 1 \mathrm{H}, \mathrm{NH}), 7.57(\mathrm{~d}$, $\left.1 \mathrm{H}, \mathrm{ArH}, J_{o}=7.20 \mathrm{~Hz}\right), 7.50-7.47(\mathrm{~m}, 2 \mathrm{H}, \mathrm{ArH}), 7.44(\mathrm{~d}$, $\left.1 \mathrm{H}, \mathrm{ArH}, J_{o}=8.80 \mathrm{~Hz}\right), 7.39-7.38(\mathrm{~m}, 1 \mathrm{H}, \mathrm{ArH}), 7.31-$ $7.27(\mathrm{~m}, 2 \mathrm{H}, \mathrm{ArH}), 7.16-7.12(\mathrm{~m}, 1 \mathrm{H}, \mathrm{ArH}), 4.62(\mathrm{q}, 2 \mathrm{H}$, $\left.\mathrm{C}_{2}-\mathrm{OCH}_{2} \mathrm{CH}_{3}, J=6.80 \mathrm{~Hz}\right), 2.97-2.93\left(\mathrm{~m}, 2 \mathrm{H}, \mathrm{CH}_{2}\right)$, 2.85-2.81 (m, 2H, CH $), 1.50$ (t, $3 \mathrm{H}, \mathrm{C}_{2}-\mathrm{OCH}_{2} \mathrm{CH}_{3}, J=$ $6.80 \mathrm{~Hz}$ ); Anal. calcd. for $\mathrm{C}_{24} \mathrm{H}_{18} \mathrm{ClN}_{3} \mathrm{O}: \mathrm{C}, 72.09 ; \mathrm{H}, 4.54$; N, 10.51; Found: C, 72.09; H, 4.54; N, 10.51\%.

$2.5 \mathrm{~h}$ 2-ethoxy-10-methyl-4-(4'-chlorophenyl)-5,6-dih ydro-11H-pyrido[2,3-a]carbazole-3-carbonitrile (6h): Yellow solid; yield: $231 \mathrm{mg}$ (56\%); M.p. $250-252{ }^{\circ} \mathrm{C}$; FTIR $\left(\mathrm{KBr}, \mathrm{cm}^{-1}\right) \nu_{\max }$ : $3267(\mathrm{NH}), 2214(\mathrm{CN}), 1552(\mathrm{C}=\mathrm{N})$; ${ }^{1} \mathrm{H}$ NMR $\left(400 \mathrm{MHz}, \mathrm{CDCl}_{3}\right)(\mathrm{ppm}) \delta_{\mathrm{H}}: 8.59$ (br s, $\left.1 \mathrm{H}, \mathrm{NH}\right)$, 7.51-7.47 (m, 2H, ArH), $7.43\left(\mathrm{~d}, 1 \mathrm{H}, \mathrm{ArH}, J_{o}=6.40 \mathrm{~Hz}\right.$ ), 7.30-7.27 (m, 2H, ArH), 7.10-7.05 (m, 2H, ArH), 4.64 (q, $\left.2 \mathrm{H}, \mathrm{C}_{2}-\mathrm{OCH}_{2} \mathrm{CH}_{3}, J=7.20 \mathrm{~Hz}\right), 2.96-2.92\left(\mathrm{~m}, 2 \mathrm{H}, \mathrm{CH}_{2}\right)$, 


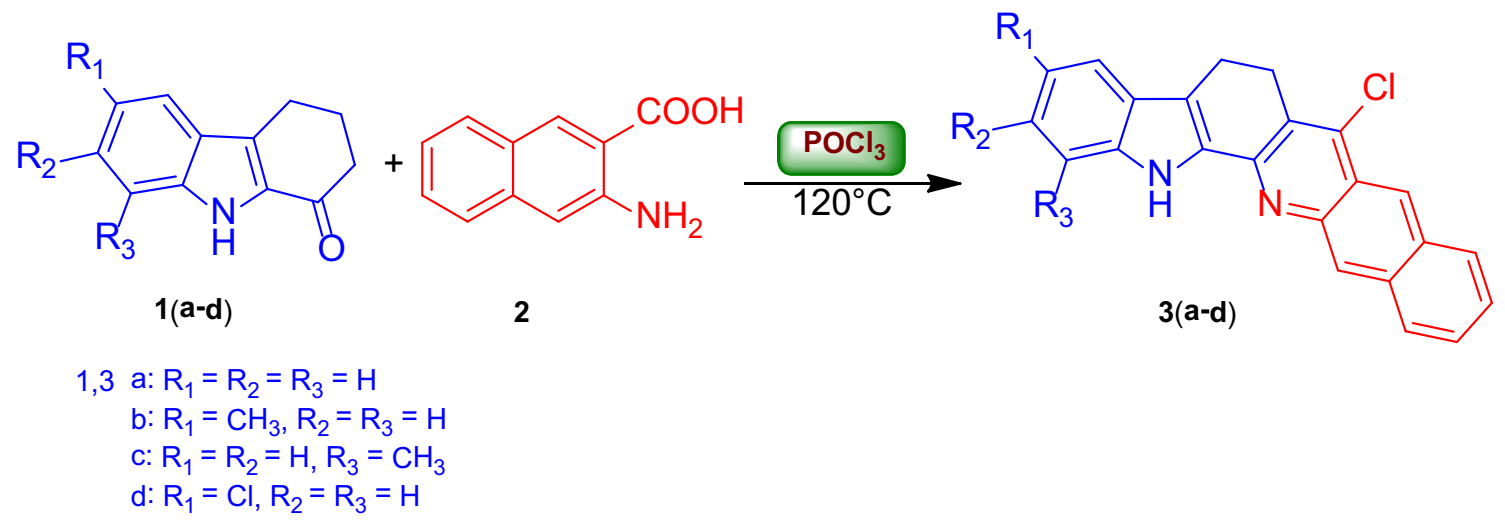

Scheme 1. Synthesis of 7-chloro-benzo[6',7'-a'] quino[2',3'-a]-5,6-dihydrocarbazoles 3.

Table 1. Scope of the modified Friedlander synthesis of Benzoquinoline-carbazole derivatives.

\begin{tabular}{lcccccc}
\hline Entry & $\mathrm{R}_{1}$ & $\mathrm{R}_{2}$ & $\mathrm{R}_{3}$ & Product & Time (h) & Yield (\%) \\
\hline 1. & $\mathrm{H}$ & $\mathrm{H}$ & $\mathrm{H}$ & 3a & 5 & 80 \\
2. & $\mathrm{CH}_{3}$ & $\mathrm{H}$ & $\mathrm{H}$ & 3b & 5 & 69 \\
3. & $\mathrm{H}$ & $\mathrm{H}$ & $\mathrm{CH}_{3}$ & $\mathbf{3 c}$ & 5.5 & 67 \\
4. & $\mathrm{Cl}$ & $\mathrm{H}$ & $\mathrm{H}$ & $\mathbf{3 d}$ & 5.5 & 55 \\
\hline
\end{tabular}

2.85-2.81 (m, 2H, $\left.\mathrm{CH}_{2}\right), 2.57\left(\mathrm{~s}, 3 \mathrm{H}, \mathrm{CH}_{3}\right), 1.50\left(\mathrm{t}, 3 \mathrm{H}, \mathrm{C}_{2}-\right.$ $\mathrm{OCH}_{2} \mathrm{CH}_{3}, J=7.20 \mathrm{~Hz}$ ); Anal. calcd. for $\mathrm{C}_{25} \mathrm{H}_{20} \mathrm{ClN}_{3} \mathrm{O}$ : C, 72.55; H, 4.87; N, 10.15; Found: C, 72.55; H, 4.87; N, $10.15 \%$.

2.5i 2-ethoxy-4-(4'-methylphenyl)-5,6-dihydro-11Hpyrido[2,3-a]carbazole-3-carbonitrile (6i): Yellow solid; yield: $333 \mathrm{mg}(88 \%)$; M.p. $263-265{ }^{\circ} \mathrm{C}$; FT-IR (KBr, $\left.\mathrm{cm}^{-1}\right) \nu_{\max }: 3336(\mathrm{NH}), 2216(\mathrm{CN}), 1555(\mathrm{C}=\mathrm{N}) ;{ }^{1} \mathrm{H}$ NMR $\left(400 \mathrm{MHz} \mathrm{CDCl}_{3}\right)(\mathrm{ppm}) \delta_{\mathrm{H}}: 8.79(\mathrm{br} \mathrm{s}, 1 \mathrm{H}, \mathrm{NH}), 7.57$ (d, $\left.1 \mathrm{H}, \mathrm{ArH}, J_{o}=8.40 \mathrm{~Hz}\right), 7.44\left(\mathrm{~d}, 1 \mathrm{H}, \mathrm{ArH}, J_{o}=8.40 \mathrm{~Hz}\right)$, $7.31\left(\mathrm{~d}, 2 \mathrm{H}, \mathrm{ArH}, J_{o}=8.00 \mathrm{~Hz}\right), 7.26-7.22(\mathrm{~m}, 2 \mathrm{H}, \mathrm{ArH})$, $7.13(\mathrm{t}, 1 \mathrm{H}, \mathrm{ArH}, J=8.00 \mathrm{~Hz}), 4.61\left(\mathrm{q}, 2 \mathrm{H}, \mathrm{C}_{2}-\mathrm{OCH}_{2} \mathrm{CH}_{3}\right.$, $J=7.20 \mathrm{~Hz}), 2.96-2.91\left(\mathrm{~m}, 2 \mathrm{H}, \mathrm{CH}_{2}\right), 2.89-2.84(\mathrm{~m}, 2 \mathrm{H}$, $\left.\mathrm{CH}_{2}\right), 2.43\left(\mathrm{~s}, 3 \mathrm{H}, \mathrm{C}_{4}{ }^{\prime}-\mathrm{CH}_{3}\right), 1.50$ (t, 3H, $\mathrm{C}_{2}-\mathrm{OCH}_{2} \mathrm{CH}_{3}$, $J=7.20 \mathrm{~Hz}) ;{ }^{13} \mathrm{C} \mathrm{NMR}\left(100 \mathrm{MHz}, \mathrm{CDCl}_{3}\right)(\mathrm{ppm}) \delta_{\mathrm{C}}$ : $163.4,154.4,148.5,138.9,137.8,132.5,132.3,129.3,128.3$, 126.7, 124.5, 121.4, 120.2, 119.8, 118.7 (CN), 115.8, 111.7, 93.4, $62.9\left(\mathrm{OCH}_{2} \mathrm{CH}_{3}\right), 25.2,21.36,19.4\left(\mathrm{C}_{4}{ }^{\prime}-\mathrm{CH}_{3}\right), 14.5$ $\left(\mathrm{OCH}_{2} \mathrm{CH}_{3}\right)$; Anal. calcd. for $\mathrm{C}_{25} \mathrm{H}_{21} \mathrm{~N}_{3} \mathrm{O}: \mathrm{C}, 79.13 ; \mathrm{H}, 5.58$; N, 11.07; Found: C, 79.22; H, 5.54; N, 11.14\%.

\section{$2.5 \mathrm{j}$ 2-ethoxy-10-methyl-4-(4'-methylphenyl)-5,6-di} hydro-11H-pyrido[2,3-a]carbazole-3-carbonitrile (6j): Yellow solid; yield: $337 \mathrm{mg}$ (86\%); M.p. $261-263{ }^{\circ} \mathrm{C}$; FTIR $\left(\mathrm{KBr}, \mathrm{cm}^{-1}\right) v_{\max }$ : $3355(\mathrm{NH}), 2214(\mathrm{CN}), 1554(\mathrm{C}=\mathrm{N})$; ${ }^{1} \mathrm{H}$ NMR $\left(400 \mathrm{MHz}, \mathrm{CDCl}_{3}\right)(\mathrm{ppm}) \delta_{\mathrm{H}}: 8.58(\mathrm{br} \mathrm{s}, 1 \mathrm{H}, \mathrm{NH})$, 7.43-7.40 (m, 1H, ArH), $7.31\left(\mathrm{~d}, 2 \mathrm{H}, \mathrm{ArH}, J_{o}=7.60 \mathrm{~Hz}\right)$, 7.25-7.22 (m, 2H, ArH), 7.08-7.04 (m, 2H, ArH), 4.63 (q, $\left.2 \mathrm{H}, \mathrm{C}_{2}-\mathrm{OCH}_{2} \mathrm{CH}_{3}, J=6.80 \mathrm{~Hz}\right), 2.94-2.92\left(\mathrm{~m}, 2 \mathrm{H}, \mathrm{CH}_{2}\right)$,
2.90-2.84 (m, 2H, $\left.\mathrm{CH}_{2}\right), 2.57$ (s, 3H, $\left.\mathrm{CH}_{3}\right), 2.42$ (s, 3H, C ' $^{\text {- }}$ $\left.\mathrm{CH}_{3}\right), 1.50\left(\mathrm{t}, 3 \mathrm{H}, \mathrm{C}_{2}-\mathrm{OCH}_{2} \mathrm{CH}_{3}, J=6.80 \mathrm{~Hz}\right)$; Anal. calcd. for $\mathrm{C}_{26} \mathrm{H}_{23} \mathrm{~N}_{3} \mathrm{O}$ : C, 79.36; H, 5.89; N, 10.68; Found: C, 79.25; $\mathrm{H}, 5.84 ; \mathrm{N}, 10.72 \%$.

$2.5 \mathrm{k}$ 2-ethoxy-10-methyl-4-(4'-methoxyphenyl)-5,6-d ihydro-11H-pyrido[2,3-a] carbazole-3 -carbonitrile (6k): Yellow solid; yield: $290 \mathrm{mg}$ (71\%); M.p. 256-258 ${ }^{\circ} \mathrm{C}$; FT-IR $\left(\mathrm{KBr}, \mathrm{cm}^{-1}\right) v_{\max }: 3299(\mathrm{NH}), 2215(\mathrm{CN}), 1640$ $(\mathrm{C}=\mathrm{N}) ;{ }^{1} \mathrm{H} \mathrm{NMR}\left(400 \mathrm{MHz}, \mathrm{CDCl}_{3}\right)(\mathrm{ppm}) \delta_{\mathrm{H}}: 8.58(\mathrm{br}$ s, 1H, NH), 7.43-7.41 (m, 1H, ArH), $7.28(\mathrm{~d} \mathrm{~d}, 2 \mathrm{H}, \mathrm{ArH}$, $\left.J_{m}=2.00 \mathrm{~Hz} \& J_{o}=6.80 \mathrm{~Hz}\right), 7.12-7.06(\mathrm{~m}, 2 \mathrm{H}, \mathrm{ArH})$, $7.03\left(\mathrm{~d} \mathrm{~d}, 2 \mathrm{H}, \mathrm{ArH}, J_{m}=2.00 \mathrm{~Hz} \& J_{o}=6.80 \mathrm{~Hz}\right), 4.63(\mathrm{q}$, $\left.2 \mathrm{H}, \mathrm{C}_{2}-\mathrm{OCH}_{2} \mathrm{CH}_{3}, J=6.80 \mathrm{~Hz}\right), 3.87\left(\mathrm{~s}, 3 \mathrm{H}, \mathrm{C}_{4}{ }^{\prime}-\mathrm{OCH}_{3}\right)$, 2.93-2.89 (m, 4H, $\mathrm{CH}_{2}$ ), 2.57 (s, $\left.3 \mathrm{H}, \mathrm{CH}_{3}\right), 1.51$ (t, 3H, $\mathrm{C}_{2}-$ $\mathrm{OCH}_{2} \mathrm{CH}_{3}, J=6.80 \mathrm{~Hz}$ ); Anal. calcd. for $\mathrm{C}_{26} \mathrm{H}_{23} \mathrm{~N}_{3} \mathrm{O}_{2}$ : C, 76.26; H, 5.66; N, 10.26; Found: C, 76.34; H, 5.64; N, $10.33 \%$.

2.51 2-Methoxy-4-(9-ethyl-9H-carbazol-3-yl)-10-met hyl-5,6-dihydro-11H-pyrido[2,3-a]carbazole-3-carbon itrile (7a): Yellow solid; yield: $318 \mathrm{mg}$ (68\%); M.p. 263-265 ${ }^{\circ} \mathrm{C}$; FT-IR $\left(\mathrm{KBr}, \mathrm{cm}^{-1}\right) v_{\max }: 3375(\mathrm{NH}), 2216$ $(\mathrm{CN}), 1557(\mathrm{C}=\mathrm{N}) ;{ }^{1} \mathrm{H}$ NMR $\left(400 \mathrm{MHz}, \mathrm{CDCl}_{3}\right)(\mathrm{ppm}) \delta_{\mathrm{H}}$ : $8.86(\mathrm{br} \mathrm{s}, 1 \mathrm{H}, \mathrm{NH}), 8.10\left(\mathrm{~d}, 1 \mathrm{H}, \mathrm{ArH}, J_{o}=7.20 \mathrm{~Hz}\right), 8.06(\mathrm{~s}$, $\left.1 \mathrm{H}, \mathrm{ArH}, J_{\mathrm{m}}=1.20 \mathrm{~Hz}\right), 7.57\left(\mathrm{~d}, 1 \mathrm{H}, \mathrm{ArH}, J_{o}=8.00 \mathrm{~Hz}\right)$, 7.54-7.48 (m, 2H, ArH), 7.46-7.42 (m, 3H, ArH), 7.30$7.26(\mathrm{~m}, 2 \mathrm{H}, \mathrm{ArH}), 7.13\left(\mathrm{t}, 1 \mathrm{H}, \mathrm{ArH}, J_{o}=7.60 \mathrm{~Hz}\right), 4.62$ $\left(\mathrm{q}, 2 \mathrm{H}, \mathrm{N}_{9}{ }^{\prime}-\mathrm{CH}_{2} \mathrm{CH}_{3}, J=7.20 \mathrm{~Hz}\right), 4.18\left(\mathrm{~s}, 3 \mathrm{H}, \mathrm{C}_{2}-\right.$ $\left.\mathrm{OCH}_{3}\right), 2.98-2.93\left(\mathrm{~m}, 4 \mathrm{H}, \mathrm{CH}_{2}\right), 1.49$ (t, 3H, N9'- $\mathrm{CH}_{2} \mathrm{CH}_{3}$, $J=7.20 \mathrm{~Hz}) ;{ }^{13} \mathrm{C} \mathrm{NMR}\left(100 \mathrm{MHz}, \mathrm{CDCl}_{3}\right)(\mathrm{ppm}) \delta_{\mathrm{C}}: 163.7$, $155.4,148.4,140.4,140.0,137.9,132.3,126.1,126.7,126.2$, $125.7,124.5,123.6,122.7,122.1,120.7,120.6,120.2,119.3$ $(\mathrm{CN}), 119.2,118.8,111.7,108.7,108.6,54.3\left(\mathrm{OCH}_{3}\right), 37.7$ ( $\left.\mathrm{N}_{9}{ }^{\prime}-\mathrm{CH}_{2} \mathrm{CH}_{3}\right), 25.4,19.4,13.8\left(\mathrm{~N}_{9}{ }^{\prime}-\mathrm{CH}_{2} \mathbf{C H}_{3}\right)$; Anal. calcd. for $\mathrm{C}_{31} \mathrm{H}_{24} \mathrm{~N}_{4} \mathrm{O}$ : C, 79.46; H, 5.16; N, 11.96; Found: C, 79.55; H, 5.12; N, $11.89 \%$.

$2.5 \mathrm{~m} \quad$ 2-Methoxy-4-(9'-ethyl-9H-carbazol-3'-yl)-10methyl-5,6-dihydro-11H-pyrido[2,3-a]carbazole-3-car bonitrile (7b): Yellow solid; yield: $313 \mathrm{mg}(65 \%)$; M.p. 
<smiles>[R]Cc1cccc2c3c([nH]c12)C(=O)CC[C+]3C</smiles>

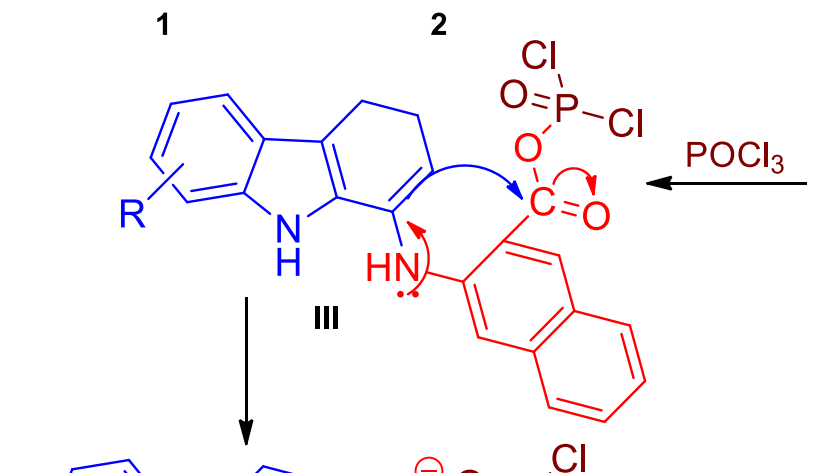

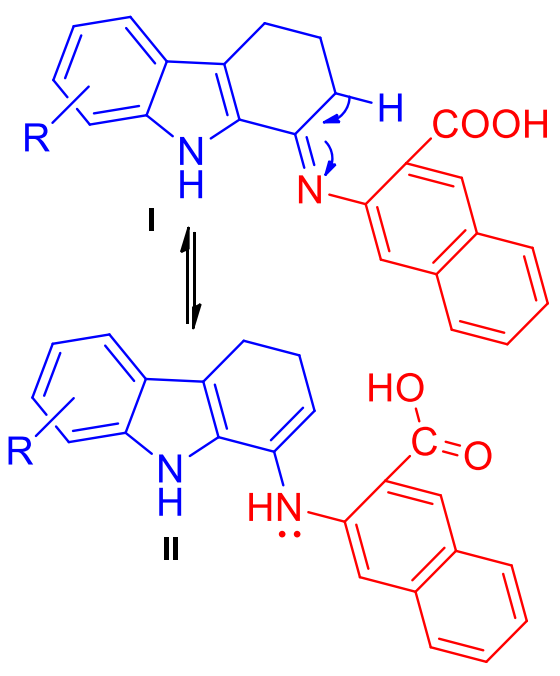

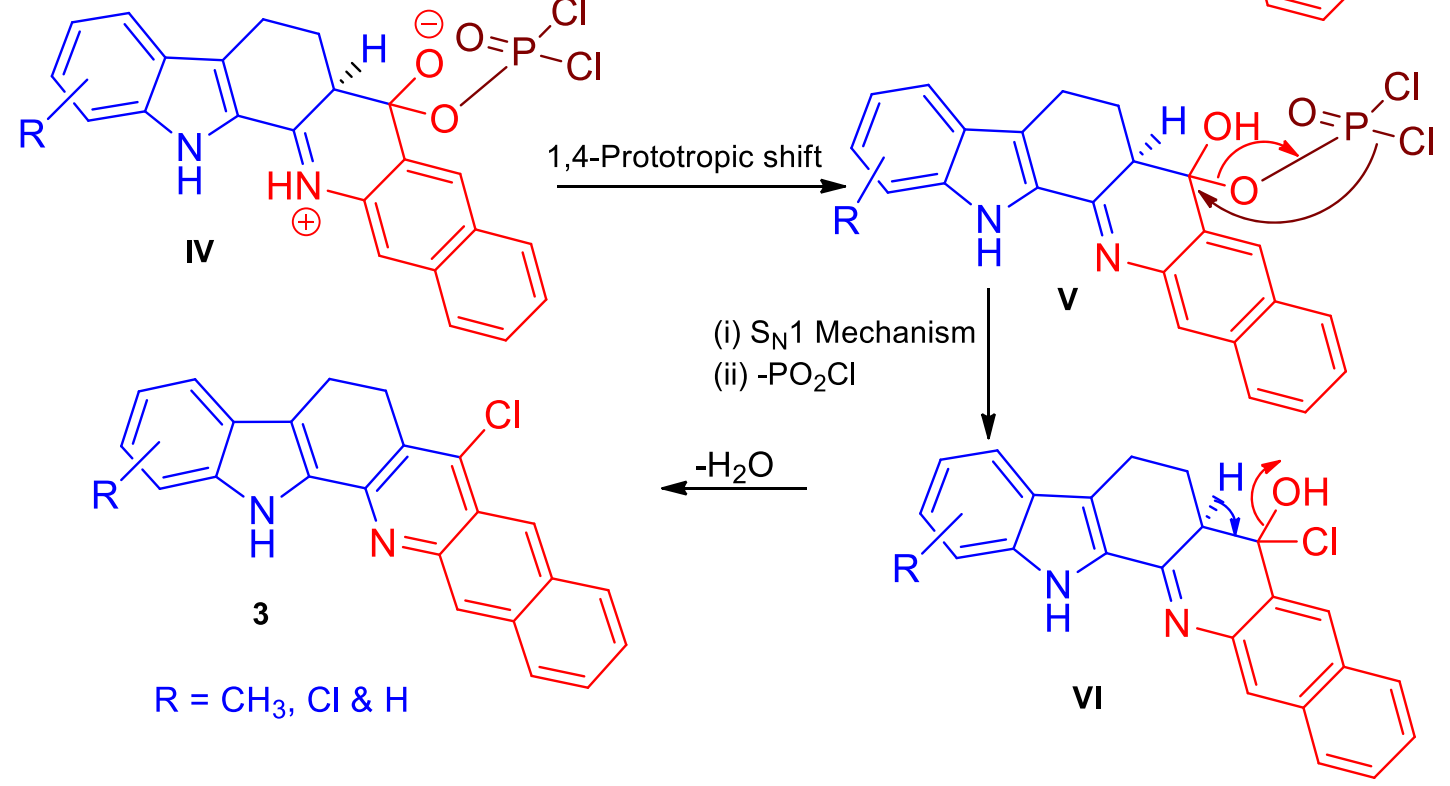

Scheme 2. Machanistic rationalization for the formation of 3.

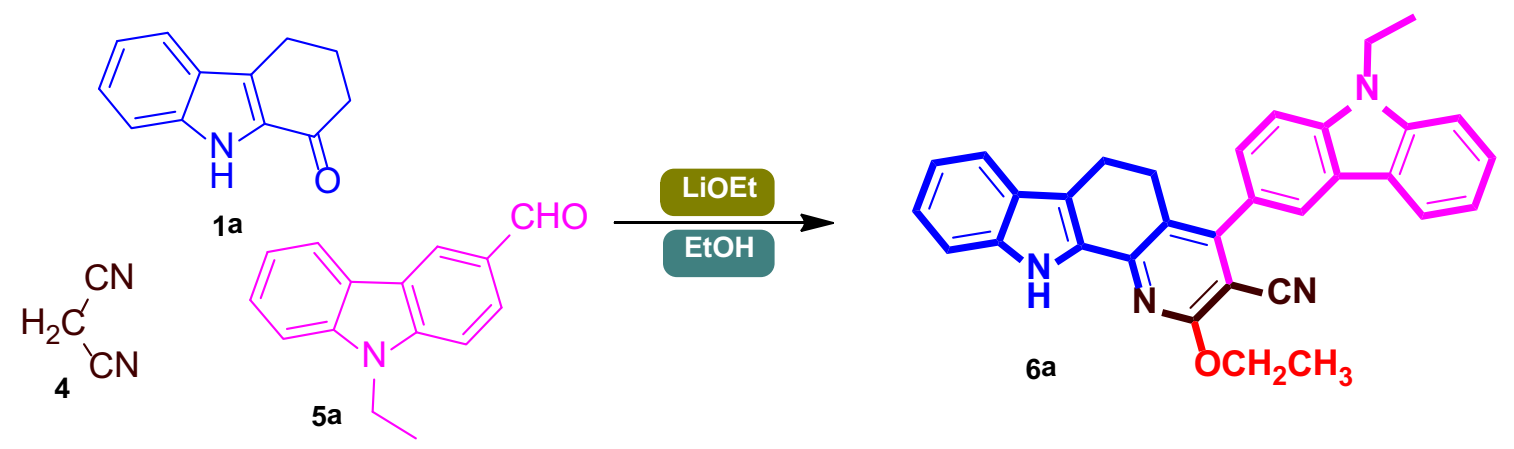

Scheme 3. Synthesis of 2-ethoxy-4-(9'-ethyl-9H-carbazol-3'-yl)-5,6-dihyro-11H-pyrido[2,3-a]carbazole3-carbonitrile $\mathbf{6 a}$.

260-262 ${ }^{\circ} \mathrm{C}$; FT-IR $\left(\mathrm{KBr}, \mathrm{cm}^{-1}\right) v_{\max }: 3391(\mathrm{NH}), 2215$ $(\mathrm{CN}), 1556(\mathrm{C}=\mathrm{N}) ;{ }^{1} \mathrm{H}$ NMR $\left(400 \mathrm{MHz}, \mathrm{CDCl}_{3}\right)(\mathrm{ppm}) \delta_{\mathrm{H}}$ : $8.75(\mathrm{br} \mathrm{s}, 1 \mathrm{H}, \mathrm{NH}), 8.10\left(\mathrm{~d}, 1 \mathrm{H}, \mathrm{ArH}, J_{o}=8.60 \mathrm{~Hz}\right), 8.58$ $(\mathrm{d}, 1 \mathrm{H}, \mathrm{ArH}, J m=1.60 \mathrm{~Hz}), 7.54-7.48(\mathrm{~m}, 2 \mathrm{H}, \mathrm{ArH}), 7.46$ (m, 2H, ArH), 7.35-7.33 (m, 2H, ArH), 7.27-7.26 (m, 1H, $\mathrm{ArH}), 7.11\left(\mathrm{~d} \mathrm{~d}, 1 \mathrm{H}, \mathrm{ArH}, J_{m}=1.60 \mathrm{~Hz} \& J_{o}=7.60 \mathrm{~Hz}\right)$, $4.42\left(\mathrm{q}, 2 \mathrm{H}, \mathrm{N}_{9}{ }^{\prime}-\mathrm{CH}_{2} \mathrm{CH}_{3}, J=7.20 \mathrm{~Hz}\right), 4.17(\mathrm{~s}, 3 \mathrm{H}$, $\left.\mathrm{C}_{2}-\mathrm{OCH}_{3}\right), 2.96-2.90\left(\mathrm{~m}, 4 \mathrm{H}, \mathrm{CH}_{2}\right), 2.44\left(\mathrm{~s}, 3 \mathrm{H}, \mathrm{CH}_{3}\right)$, 
1.49 (m, 3H, N ${ }_{9}{ }^{-}-\mathrm{CH}_{2} \mathrm{CH}_{3}$ ); Anal. calcd. for $\mathrm{C}_{32} \mathrm{H}_{26} \mathrm{~N}_{4} \mathrm{O}$ : C, 79.64; H, 5.43; N, 11.61; Found: C, 79.73; H, 5.47; N, $11.54 \%$.

\section{Results and Discussion}

The two-component Friedländer reaction of 2-aminoaryl ketones with carbonyl compounds containing a reactive $\alpha$-methylene group in order to obtain benzoquinoline compounds has been known for a long time. ${ }^{17-22}$ However, to the best of our knowledge, none of these report the synthesis of benzoquinoline-carbazole derivatives via a $\mathrm{POCl}_{3}$ promoted Friedländer reaction.

Prompted by the encouraging importance of benzoqu inoline-carbazole derivatives via Friedländer reaction, we embarked on the synthesis of 7-chloro-benzo[ $\left[6^{\prime}, 7^{\prime}\right.$ $a^{\prime}$ ]quino[2', $\left.3^{\prime}-a\right]$-5,6-dihydrocarbazole 3 by a modified Friedländer hetero-annulation reaction of 2,3,4,9tetrahydrocarbazol-1-one 1 with 3-amino-2-naphthoic acid 2 using catalytic amounts of $\mathrm{POCl}_{3}$ under solventfree condition (Scheme 1). All these reactions were carried out by refluxing an equimolar ratio of the reactants at $120^{\circ} \mathrm{C}$.

Next, we examined the scope of the transformation utilizing the reactivity of different substituted 2,3,4,9tetrahydrocarbazol-1-ones $\mathbf{1}$ (a-d) as substrates; all of the reactions afforded the corresponding benzoquino line-carbazole products $\mathbf{3}$ (a-d) in moderate to good yields (Table 1).

The proposed structures of the synthesized compounds were consistent with their FT-IR, ${ }^{1} \mathrm{H}$ NMR, ${ }^{13} \mathrm{C}$ NMR spectra and elemental analyses. The FT-IR spectral data of 3a displayed prominent absorption peaks at 3436 and $1592 \mathrm{~cm}^{-1}$ due to indole $\mathrm{NH}$ and $\mathrm{C}=\mathrm{N}$ stretchings respectively. The ${ }^{1} \mathrm{H}$ NMR spectrum of $\mathbf{3 a}$ exhibited a broad singlet for the indole $\mathrm{NH}$ at $\delta 9.47 \mathrm{ppm}$. Two singlets at $\delta 8.66$ and $\delta 8.52 \mathrm{ppm}$ assigned to $\mathrm{C}_{8}$ and $\mathrm{C}_{13}$ protons. The two multiplet signals in the region of $\delta$ 8.04-7.98 ppm due to $\mathrm{C}_{12} \& \mathrm{C}_{9}$ protons and $\delta 7.52-7.47$ ppm were attributed to the $\mathrm{C}_{11} \& \mathrm{C}_{10}$ aromatic protons respectively. The signal due to $\mathrm{C}_{4}$ proton occurred as a doublet at $\delta 7.62\left(J_{o}=7.60 \mathrm{~Hz}\right) \mathrm{ppm}$ and a doublet at $\delta$ $7.39\left(J_{o}=8.40 \mathrm{~Hz}\right)$ arising from the $\mathrm{C}_{1}$ proton. A multiplet at $\delta$ 7.29-7.27 ppm was assigned to a $\mathrm{C}_{3}$ proton, a triplet at $\delta 7.13\left(J_{o}=7.20 \mathrm{~Hz}\right)$ was assigned to the $\mathrm{C}_{2}$ proton while the aliphatic protons $\left(\mathrm{C}_{6}\right.$ and $\left.\mathrm{C}_{5}\right)$ resonated as multiplets centered at $\delta 3.50 \mathrm{ppm}$ and $3.19 \mathrm{ppm}$ respectively. The ${ }^{13} \mathrm{C}$ NMR spectrum of $\mathbf{3 a}$ displayed 23 resonances in agreement with the proposed structure.

The plausible mechanism for the formation of compound $\mathbf{3}$ has been shown in Scheme 2 as reported earlier by our research group. ${ }^{23}$ Initially, 2,3,4,9-tetrahydrocar
Table 2. Screening of catalyst for the multicomponent synthesis of $\mathbf{6} \mathbf{a}^{\mathrm{a}}$.

\begin{tabular}{lcccc}
\hline Entry & Solvent & Catalyst (equiv) & Time & Yield $^{\mathrm{b}}$ \\
\hline 1. & EtOH & Catalyst-free & 12 & $-{ }^{\mathrm{c}}$ \\
2. & EtOH & $\mathrm{NaOH}_{2}$ & 8 & 20 \\
3. & EtOH & $\mathrm{K}_{2} \mathrm{CO}_{3}$ & 8 & 29 \\
4. & EtOH & $\mathrm{Et}_{3} \mathrm{~N}$ & 6 & 57 \\
5. & EtOH & DABCO & 6 & 31 \\
6. & EtOH & Morpholine & 6 & 27 \\
7. & EtOH & Piperidine & 5 & 38 \\
8. & EtOH & NaOEt (1) & 5 & 47 \\
9. & EtOH & LiOEt (1) & 3 & 58 \\
10. & EtOH & LiOEt (1.5) & 3 & 61 \\
11. & EtOH & LiOEt (2) & 3 & 68 \\
12. & EtOH & LiOEt (3) & 3 & 73 \\
13. & EtOH & LiOEt (4) & 6 & 67 \\
\hline
\end{tabular}

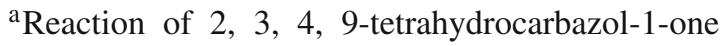
$1 a(1.0 \mathrm{mmol})$, malononitrile $4(1.0 \mathrm{mmol})$ and 9-ethyl-3-carbazolecarboxaldehyde 5a $(1.0 \mathrm{mmol})$ using LiOEt ( 3 equiv) under EtOH.

${ }^{\mathrm{b}}$ Isolated yields, ${ }^{\mathrm{c}}$ Trace product.

bazol-1-one 1 is condensed with 3-amino-2-naphthoic acid $\mathbf{2}$ in the presence of an acid $\mathrm{POCl}_{3}$ as a catalyst to afford the stable geometrical isomer $\mathbf{E}$ as intermediate I. Upon tautomerisation the more stable imine intermediate I gave an enamine intermediate II. The addition of $\mathrm{POCl}_{3}$ to the carboxylic acid intermediate II causes the formation of a mixed anhydride intermediate III, which then underwent an intramolecular electrophilic substitution reaction to yield the intermediate IV and this intermediate on subsequent 1,4-prototropic shift followed by an $\mathrm{S}_{\mathrm{N}} 1$ reaction cum $\mathrm{PO}_{2} \mathrm{Cl}$ elimination afforded the intermediate VI. The trans dehydration of intermediate VI gave the hybrid compound, 7-chlorobenzo[6 $\left.6^{\prime}, 7^{\prime}-a^{\prime}\right]$ quino[2', $\left.3^{\prime}-a\right]$-5,6-dihydrocarbazole 3 .

9-Ethyl-3-carbazolecarboxaldehyde derivatives have been utilized as versatile coupling components in the preparation of a number of nitrogen-containing heteroaromatics. ${ }^{24}$ The simple carbazoles reported so far from our laboratory were found to have anticancer, antibacterial, antioxidant and photophysical activities. ${ }^{16}$ However, the dimerised carbazoles/carbazolyl carbazole derivatives have an electron-rich structure, high thermal stability and unique electrical and optical properties $^{25,26}$ compared to its simple carbazoles. The conjugated carbazole dimer enhanced greatly the fluorescence of carbazole. ${ }^{27}$ The importance of pyridocarbazole and carbazole dimer derivatives prompted us to design a practical and efficient multicomponent reaction for the preparation of highly functionalized carbazole substituted pyridocarbazole derivatives. Recently, our research group envisaged the possibility of pseudo 
Table 3. Scope of aryl/heteroaryl substituted pyrido $[2,3-a]$ carbazoles $6(\mathbf{a}-\mathbf{k})$.<smiles>[R]c1c2cc3c([nH]c-2c([R])c1[R])C(=O)CCC3</smiles>

multicomponent reaction for the preparation of pyridocarbazoles. ${ }^{28}$

The synthetic pathways employed to prepare the targeted derivatives are depicted in Scheme 3. In a onepot, pseudo three-component heterocyclo condensation process, 2-ethoxy-4-(9'-ethyl-9H-carbazol-3'-yl)5,6-dihyro- $11 H$-pyrido[2,3- $a$ ]carbazole-3-carbonitrile 6a was obtained via a base mediated Michael additioncyclization of 2,3,4,9-tetrahydrocarbazol-1-one 1a with malononitrile 4 and 9-ethyl-3-carbazolecarboxaldehyde 5a in refluxing EtOH. In this reaction, the solvent ethanol could act as both a reactant and a solvent.

Table 2 shows the results obtained from using different reaction conditions for the synthesis of $\mathbf{6 a}$. In order to justify the significance of base in this multicomponent process, the reaction was first performed in the absence of base wherein the reaction failed to occur even at prolonged reaction time (Table 2, entry 1 ). In the presence of sodium hydroxide or potassium carbonate, the reaction occurred with isolated yields of $\mathbf{6 a}$ after $8 \mathrm{~h}$ of $20 \%$ and 


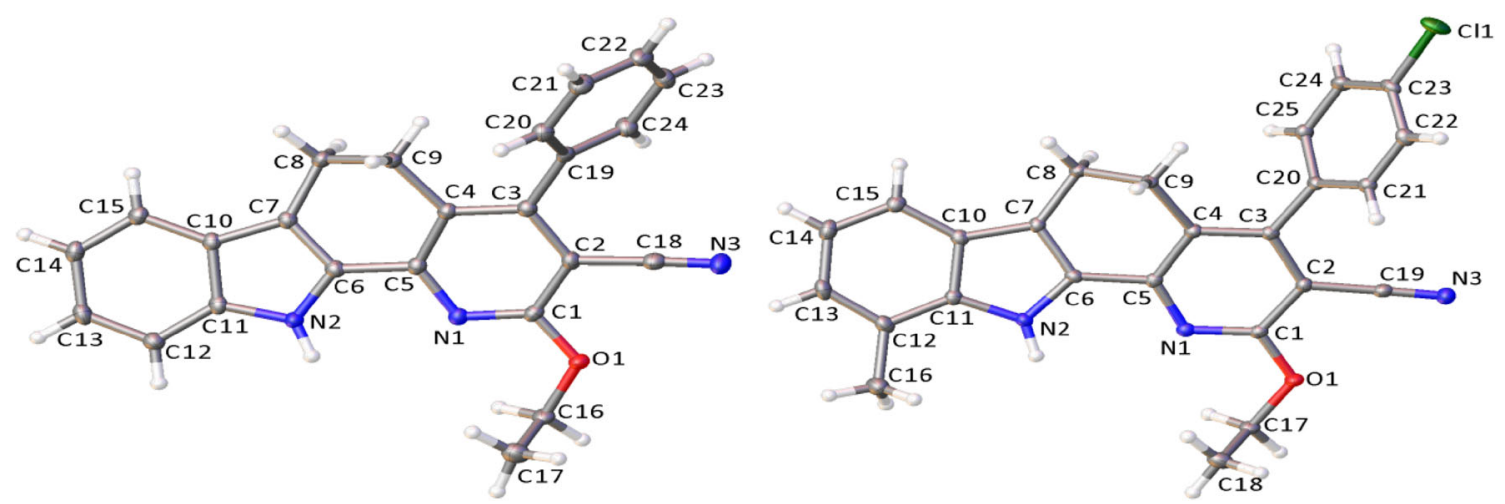

Figure 1. Illustration of the structures of $\mathbf{6 c}$ (left) and $\mathbf{6 h}$ (right) (only one unique molecule shown). Anisotropic displacement parameters are depicted at the $50 \%$ probability level.

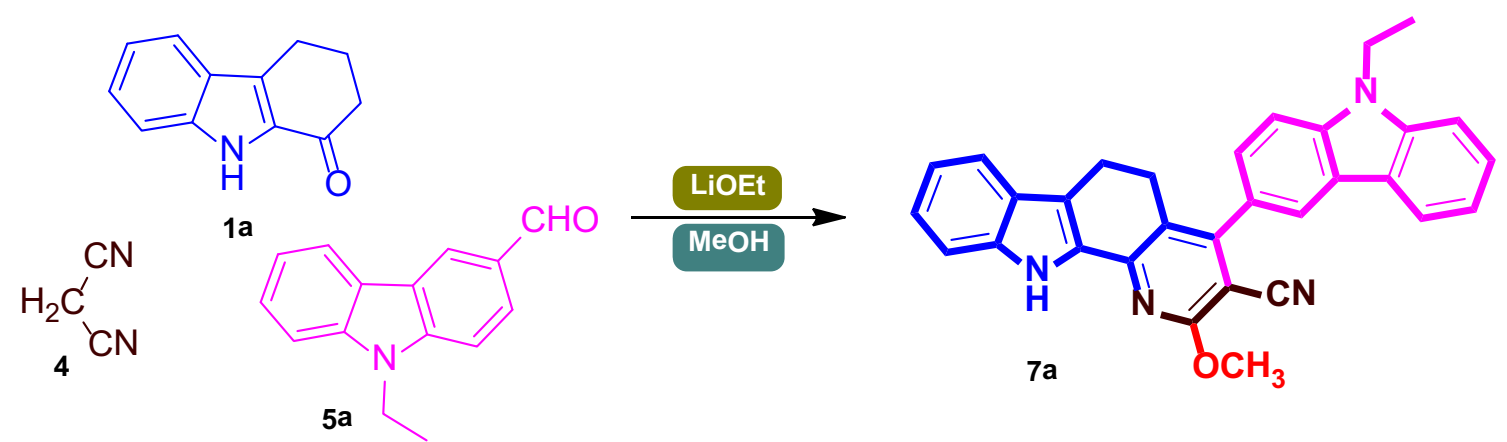

Scheme 4. Synthesis of 2-methoxy-4-(9'-ethyl-9H-carbazol-3'-yl)-5,6-dihyro-11H-pyrido[2,3-a]carbazole3-carbonitrile 7a.

$29 \%$ respectively (Table 2, entries 2 and 3). Carrying out the reaction in the presence of triethylamine (Table 2, entry 4 ) for $6 \mathrm{~h}$ led to a significant increase in the yield of $6 \mathbf{a}$. The reaction was then performed with bases such as DABCO, morpholine and piperidine (Table 2, entries 5-7), however, these bases were not as effective and resulted in low yields $(<38 \%)$ after 5-6 h. Our research group has previously reported the use of NaOEt as a base to afford simple pyridocarbazoles. ${ }^{28}$ However, in our present work, the use of $\mathrm{NaOEt}$ as a catalyst yielded only $47 \%$ of carbazole compound (Table 2, entry 8 ). Interestingly, the use of one equivalent of LiOEt instead of NaOEt increases the product yield up to $58 \%$ after $3 \mathrm{~h}$ (Table 2, entry 9). The optimum quantity of LiOEt required was screened and it was found that on increasing the amount of catalyst from 1.0 to 3.0 equiv, the yield of the reaction increased gradually but beyond 3.0 equiv, there was no significant improvement in the rate or yield of the reaction even at prolonged time (Table 2, entries 9-13). From the results, LiOEt was found to be the optimum catalyst for this transformation, wherein $73 \%$ of product $\mathbf{6 a}$ was obtained in $3 \mathrm{~h}$. The literature survey revealed the catalytic potential of LiOEt in many MCRs. ${ }^{29}$
Based on the appropriate reaction conditions, a series of 2-ethoxy-4-aryl/heteroaryl-5, 11-dihyro-6 $\mathrm{H}$ pyrido $[2,3-a$ ] carbazole-3-carbonitrile derivatives 6 $(\mathbf{a}-\mathbf{k})$ were synthesized. The results are summarized in Table 3.

The structures of compounds $\mathbf{6}(\mathbf{a}-\mathbf{k})$ were established on the basis of their elemental analyses and spectral data. The important diagnostic bands in the FT-IR spectrum of $\mathbf{6 a}$ were assigned, with the stretching vibrations at 3259 and $1544 \mathrm{~cm}^{-1}$ corresponding to indole $\mathrm{NH}$ and $\mathrm{C}=\mathrm{N}$ groups respectively and the cyano group stretching vibration assigned to a sharp band at $2216 \mathrm{~cm}^{-1}$. The ${ }^{1} \mathrm{H}$ NMR spectrum displayed a broad singlet at $\delta 8.81$ ppm attributed to the indole $\mathrm{NH}$ proton while the signal from $\mathrm{C}_{5}$ ' $-\mathrm{H}$ was visible as a doublet at $\delta 8.10\left(J_{o}=\right.$ $8.00 \mathrm{~Hz}$ ) and a sharp singlet at $\delta 8.06 \mathrm{ppm}$ was assigned to $\mathrm{C}_{4}$ ' proton. The six aromatic protons at $\mathrm{C}_{8}{ }^{\prime}, \mathrm{C}_{2}{ }^{\prime}, \mathrm{C}_{1}$ ', $\mathrm{C}_{7}, \mathrm{C}_{10}$ and $\mathrm{C}_{7}$ positions resonated as multiplets in the region of $\delta 7.58-7.44 \mathrm{ppm}$ and the $\mathrm{C}_{6}$ ' aromatic proton appeared as a multiplet at $\delta 7.29-7.27 \mathrm{ppm}$. The $\mathrm{C}_{9}$ and $\mathrm{C}_{8}$ protons were visible as a multiplet at $\delta 7.15-7.11$ ppm, the $\mathrm{OCH}_{2} \mathrm{CH}_{3}$ protons appeared as a quartet at $\delta 4.64(J=7.20 \mathrm{~Hz})$. The two protons of $\mathrm{N}_{9}{ }^{\prime}-\mathrm{CH}_{2}$ appeared as a quartet at $\delta 4.42 \operatorname{ppm}(J=7.20 \mathrm{~Hz})$ 


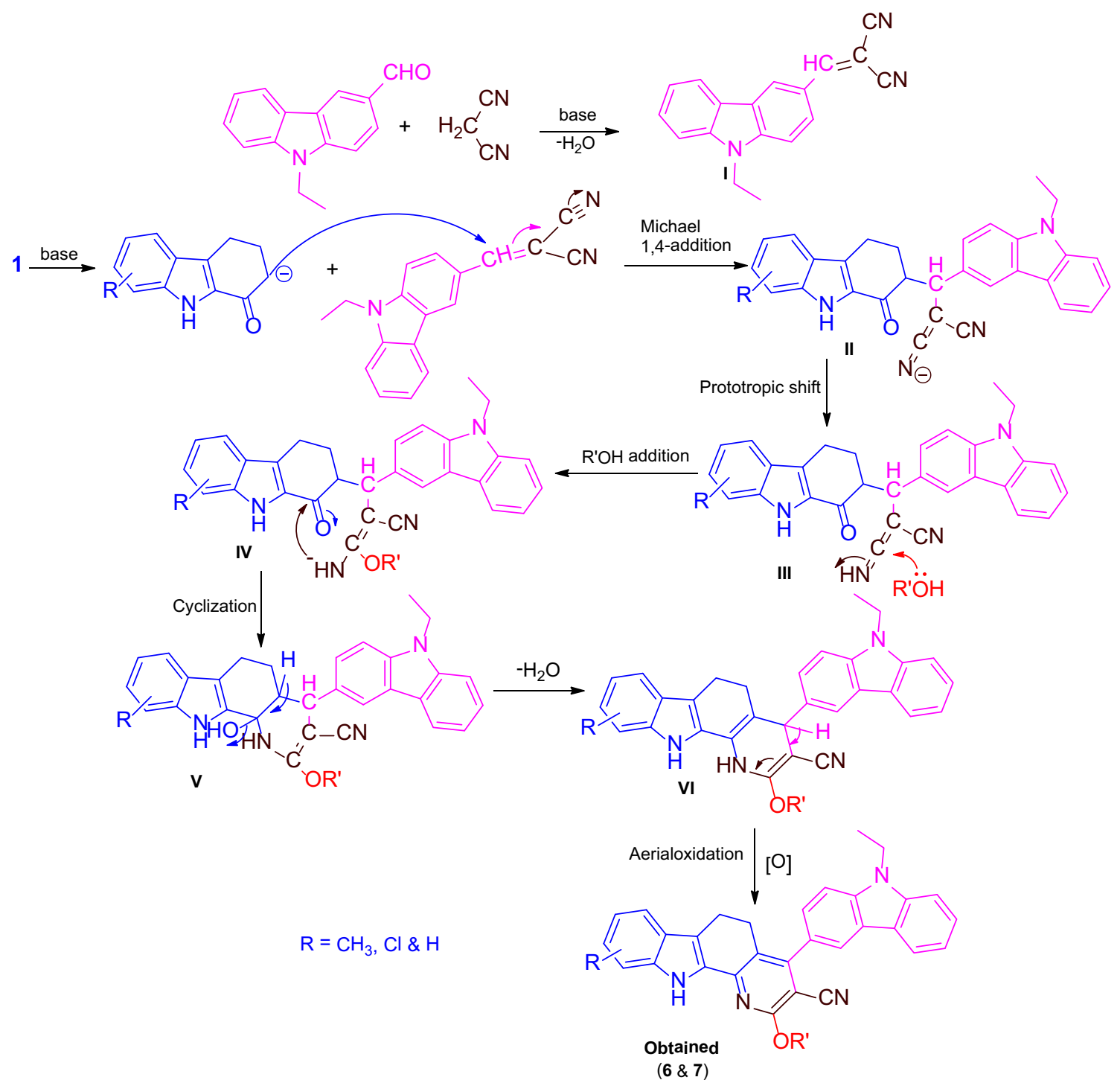

Scheme 5. Machanistic rationalization for the formation of $6 \& 7$.

while the methylene protons of $\mathrm{C}_{5}$ and $\mathrm{C}_{6}$ resonated as a multiplet at $\delta 2.97-2.94 \mathrm{ppm}$. Six methyl protons of $\mathrm{OCH}_{2} \mathrm{CH}_{3}$ and $\mathrm{N}_{9}{ }^{\prime}-\mathrm{CH}_{2} \mathrm{CH}_{3}$ appeared as a multiplet at $\delta$ 1.54-1.48 ppm. The ${ }^{13} \mathrm{C}$ NMR spectrum of 6a displayed 32 resonances in agreement with the proposed structure. The resonance signals at $\delta 63.1,37.7,14.6$ and 13.8 were attributed to $\mathrm{OCH}_{2} \mathrm{CH}_{3}, \mathrm{~N}_{9}{ }^{\prime}-\mathrm{CH}_{2} \mathrm{CH}_{3}, \mathrm{OCH}_{2} \mathbf{C H}_{3}$ and $\mathrm{N}_{9}$ ' $-\mathrm{CH}_{2} \mathbf{C H}_{3}$ carbons. The structures of $\mathbf{6 c}$ and 6h were further confirmed by single X-ray diffraction studies (Figure 1).

After the successful synthesis of 2-ethoxy-4-(9'ethyl-9H-carbazol-3'-yl)-5,6-dihyro-11 $H$-pyrido[2,3$a$ ] carbazole-3-carbonitrile $\mathbf{6 a}$, this catalytic system was used for the synthesis of 2-methoxy-4-(9'-ethyl-9Hcarbazol-3'-yl)-5,6-dihyro-11 $H$-pyrido[2,3- $a$ ] carbazo le-3-carbonitrile $7 \mathbf{a}$ by the condensation of same reactants in the presence of $\mathrm{MeOH}$ instead of $\mathrm{EtOH}$ as solvent (Scheme 4).
The analysis of the ${ }^{1} \mathrm{H}$ NMR and ${ }^{13} \mathrm{C}$ NMR data of the products led us to conclude that, when $\mathrm{MeOH}$ or EtOH was used as a solvent the reaction mechanism changed from a three-component to a four-component pathway, as depicted in Scheme 5. Initially, the intermediate I was formed via Knoevenagel condensation of 9-ethyl-3-carbazolecarboxaldehyde with malononitrile in the presence of a base. On subsequent base promoted the 1,4-Michael addition of electron deficient Knoevenagal adduct to the carbanion, derived from the synthon, 2,3,4,9-tetrahydro- $1 H$-carbazol-1-one affords the dinitrile intermediate II, which undergoes prototropic shift and alcoholic addition facilitated by the base, LiOEt to form an intermediate IV through the intermediate III. An intramolecular cyclization of intermediate IV could furnish the intermediate $\mathbf{V}$, which upon dehydration and aerial oxidation could give rise to the product 6/7. 
Table 4. The scope of various 9-ethyl-3-carbazolecarboxaldehyde substituted pyrido [2, 3- $a$ ] carbazole derivatives.

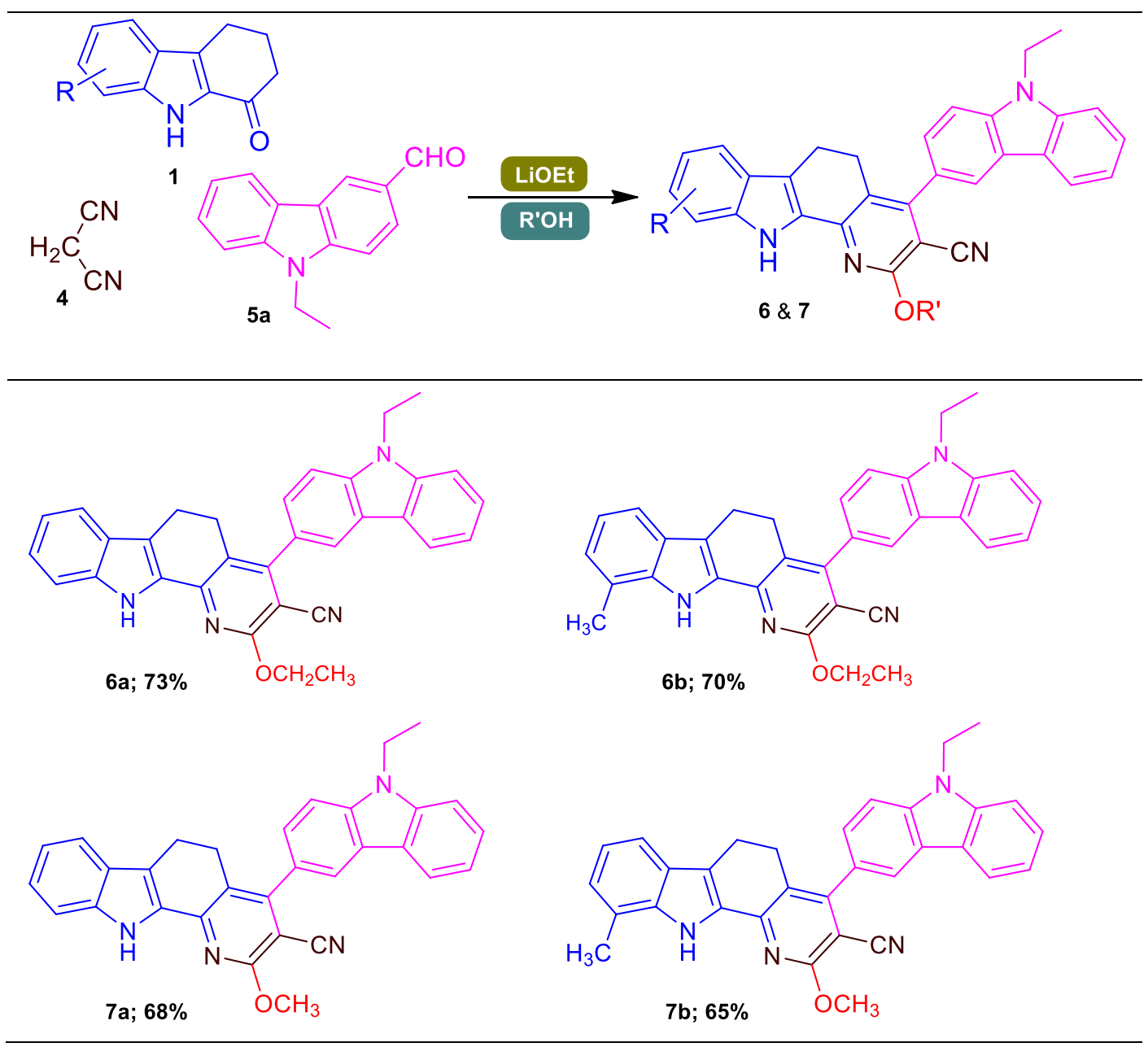

The scope and general applicability of this methodology have been investigated by using different 2,3 , 4, 9-tetrahydrocarbazol-1-ones $\mathbf{1}(\mathbf{a}, \mathbf{b})$ with 9-ethyl-3carbazolecarboxaldehyde 5a in different solvent conditions (Table 4).

\section{Conclusion}

In conclusion, we have demonstrated that differently substituted 2, 3, 4, 9-tetrahydrocarbazol-1-ones and 3-amino-2-naphthoic acid undergo an efficient Friedlander condensation reaction in the presence of $\mathrm{POCl}_{3}$ to yield benzoquinoline-carbazoles. The synthon, 2, 3, 4, 9-tetrahydrocarbazol-1-ones further undergo a pseudo three-component reaction with malononitrile and 9ethyl-3-carbazole carboxaldehyde to afford a carbazole engrafted pyridocarbazole derivatives. The investigation on solvent effect on this reaction procedure revealed that solvent could change reaction mechanism from threecomponent to four-component pathway. On the whole, the methods presented here are significant in terms of good yields, short reaction time, cost-effectiveness, readily available substrates and wide scope for production of a diversity of the products and potentially bioactive compounds.

\section{Supplementary Information (SI)}

All additional information pertaining to characterization of the compounds using ${ }^{1} \mathrm{H}$ NMR and ${ }^{13} \mathrm{C}$ NMR spectra (Figures S1-S21) and table of Crystal data are given in the supporting information.

\section{Acknowledgements}

We would like to thank the University of Mysore, for NMR data. X-ray diffraction was funded by University of Bristol, United Kingdom. Financial support from "UGC-Emeritus fellowship" [Award NO.F.6-6/2015-17/EMERITUS-2015-17OBC-7410/ (SAII)] for research, is gratefully acknowledged by Prof. K. J. Rajendra Prasad. Arya. K. R acknowledges the award of BSR-Senior Research Fellowship by University Grant Commission (UGC), New Delhi. 


\section{References}

1. Suresh J R, Syam Kumar U K, Ila H and Junjappa H 2001 Aromatic and heteroaromatic annelation studies on 3[bis(methylthio)methylene]-1-methyloxindole: synthesis of carbazoles and an efficient route to pyrido[2,3b]indoles Tetrahedron 57781

2. Hu L X, Li Z R, Wang Y M, Wu Y B, Jiang J D and Boykin D W 2007 Novel pyridinyl and pyrimidinylcarbazole sulfonamides as antiproliferative agents Bioorg. Med. Chem. Lett. 171193

3. Dračínský M, Sejbal J, Rygerová B and Stiborová M 2007 An efficient modification of ellipticine synthesis and preparation of 13-hydroxyellipticine Tetrahedron Lett. 486893

4. Barbieri V and Ferlin M G 2006 Microwave-assisted one-pot synthesis of substituted tetrahydrocarbazole and 8,9,10,11-tetrahydro-7H-pyrido[a]carbazoles Tetrahedron Lett. 478289

5. Haider N, Jabara R, Khadami F and Wanko R 1998 Synthesis of Pyridazino [4, 5- $b$ ] carbazoles as potential antitumor agents Heterocycles 481609

6. Hedin V M, Tabka T, Poulain L, Godard T, Lachevrel M, Saturnino C, Lancelot J C, Le Talaer J Y and Gauduchon P 2000 Biological properties of 5,11-dimethyl6H-pyrido [3, 2-b] carbazoles: a new class of potent antitumor drugs Anticancer Drug Des. 15109

7. de Koning C B, Michael J P and Rosseau A L 2000 A versatile and convenient method for the synthesis of substituted benzo[a]carbazoles and pyrido[2,3-a]carbazoles J. Chem. Soc. Perkin Trans. 11705

8. Hirata K, Ito C, Furukawa H, Itogiawa M, Mark Cosentino L and Lee K H 1999 Substituted 7Hpyrido[4,3-c]carbazoles with potent anti-HIV activity Bioorg. Med. Chem. Lett. 9119

9. Ohashi M, Shudo T, Nishijima K, Notso T, Kikuchi A, Yanagibashi K and Nishida H 2000 Pyridocarbazole derivatives having cGMP-PDE inhibitory activity, United States Patent 6018046 United States Mochida Pharmaceutical Co., Ltd, Tokyo, JP

10. Selvi G and Rajendran S P 2004 Synthesis of some new 2-[3-(2-chloro quinolinyl)]-3-aryl-4-thiazolidinones as potent antibacterial agents J. Asian Chem. 161017

11. Carr B A and Franklin M R 1998 Drugmetabolizing enzyme induction by $2,2^{\prime}$-dipyridyl, 1,7-phenanthroline,7,8-benzoquinoline and oltipraz in mouse Xenobiotica 28949

12. Mikhailitsyn F S, Kozyreva N P, Rabinovich S A, Maksakovskaya Y V, Kulikovskaya I M, Dadasheva N R, Lebedeva M N, Bekhli A F, Lychko N D and Uvarova N A 1992 Search for new antiparasitic agents, Synthesis, toxicity, and antimalarial effect of some nitrogen-containing heterocycles with 4-(4-alkylpiperazin-1-yl)phenylamino substituents Med. Parazitol. Parazit. Bolezni. 50

13. Nozulak J, Vigouret J M, Jaton A L, Hofmann A, Dravid A R, Weber H P, Kalkman H O and Walkinshaw M D 1992 Centrally acting al-adrenoceptor agonists based on hexahydronaphth[2,3-b]-1,4-oxazines and octahydrobenzo[g]quinolinesl J. Med. Chem. 35480

14. Szmuszkovicz J, Darlington W H and Von Voigtlander P F 1988 Preparation and formulation of antipsychotic aminopolyhydrobenz(iso)quinolines and intermediates. WO 8804292 A1 1988 Chem. Abstr. 11075335

15. Kantevari S, Yempala T, Surineni G, Sridhar B, Yogeeswari P and Sriram D 2011 Synthesis and antitubercular evaluation of novel dibenzo[b,d]furan and 9-methyl-9H-carbazole derived hexahydro-2Hpyrano[3,2-c]quinolines via Povarov reaction Eur. J. Med. Chem. 464827

16. (a) Prabha K and Rajendra Prasad K J 2016 Synthesis and Cytotoxic Distinction of Benzo[h]naphtho[1,2-b] [1,6] Naphthyridine and its Isomeric Benzo[b]naphtho[1,2h][1,6] Naphthyridines Med. Chem. 62 062; (b) Indumathi T, Muthusankar A, Shanmughavel P and Rajendra Prasad K J 2013 Synthesis of hetero annulated carbazoles: exploration of in vitro cytotoxicity and molecular docking studies Med. Chem. Commun. 4 450; (c) Indumathi T, Jamal Ahamed V S, SurkSik Moon, Fronczek F R and Rajendra Prasad K J 2011 L-Proline anchored multicomponent synthesis of novel pyrido[2,3-a]carbazoles; investigation of in vitro antimicrobial, antioxidant, cytotoxicity and structure activity relationship studies Eur. J. Med. Chem. 46 5580; (d) Murali K, Sparkes H A and Rajendra Prasad K J 2017 Synthesis of hetero annulated isoxazolo-, pyrido- and pyrimido carbazoles: Screened for in vitro antitumor activity and structure activity relationships, a novel 2-amino-4-(3'-bromo-4'-methoxyphenyl)-8chloro-11H-pyrimido[4,5- $a$ ]carbazole as an antitumor agent Eur. J. Med. Chem. 128 319; (e) Yamuna E, Yurcho A, Sovesky R J, Smith P M, Zeller M and Rajendra Prasad K J 2011 Elegant one-pot synthesis of quinolino[2', 3' : 7,6]-cyclohept[1,2-b]indole through Friedländer and Pfitzinger annulation reaction Synth. Commun. 413351

17. (a) Ryabukhin S V, Naumchik V S, Plaskon A S, Grygorenko O O and Tolmachev A A 2011 3Haloquinolines by Friedländer reaction of r-haloketones J. Org. Chem. 76 5774; (b) Le Z-G, Liang M, Chen Z-S, Zhang S-H and Xie Z-B 2017 Ionic liquid as an efficient medium for the synthesis of quinoline derivatives via $\alpha$-chymotrypsin catalysed Friedländer condensation Molecules 22762 ; (c) Tufail F, Saquib M, Singh S, Tiwari J, Singh M, Singh J and Singh J 2017 Bioorganopromoted green Friedländer synthesis: a versatile new malic acid promoted solvent free approach to multisubstituted quinoline New J. Chem. 411618

18. (a) Zhang X-L, Wang Q-Y, Sheng S-R, Wang Q and Liu X-L 2009 Efficient Friedländer Synthesis of quinoline derivatives from 2-aminoarylketones and carbonyl compounds mediated by recyclable PEG-supported sulfonic acid Synth. Commun. 39 3293; (b) Ramann G A and Cowen B J 2016 Recent advances in metalfree quinoline synthesis Molecules 21 986; (c) Reddy B P, Iniyavan P, Sarveswari S and Vijayakumar V 2014 Nickel oxide nanoparticles catalyzed synthesis of polysubstituted quinolines via Friedlander hetero-annulation reaction Chin. Chem. Lett. 251595

19. Luo W, Mu Q, Qiu W, Liu T, Yang F, Liu X and Tang J 2011 A novel Friedlander-type synthesis of 3aryl quinolines from 3-oxo-2,3-diaryl-propionaldehydes Tetrahedron 677090 
20. Genovese S, Epifano F, Marcotullio M C, Pelucchini C and Curini M 2011 An alternative quinoline synthesis by via Friedländer reaction catalyzed by $\mathrm{Yb}(\mathrm{OTf}) 3$ Tetrahedron Lett. 523474

21. McNaughton B R and Miller B L 2003 A mild and efficient one-step synthesis of quinolines Org. Lett. 54257

22. Satheeshkumar R, Shanker R, Kaminsky W and Rajendra Prasad K J 2016 Novel synthetic and mechanistic approach of TFA catalyzed Friedlander synthesis of 2-acylquinolines from symmetrical and unsymmetrical 1,2-diketones with o-aminoarylketone ChemistrySelect. 16823

23. Prabakaran K and Rajendra Prasad K J 2010 Simple and convenient methods for the synthesis of indolo[3,2c]acridines Synth. Commun. 403528

24. Ashok D, Ravi S, Vijaya Lakshmi B and Ganesh A 2015 One-pot synthesis of carbazole based 3-hydroxy-4Hchromen-4-ones by modified Algar-Flynn-Oyamada reaction and their antimicrobial activity J. Serb. Chem. Soc. 801361

25. (a) Song Y, Di C-A, Wei Z, Zhao T, Xu W, Liu Y, Zhang D and Zhu D 2008 Synthesis, characterization, and field-effect transistor properties of carbazolenevinylene oligomers: from linear to cyclic architectures
Chem. Eur. J. 14 4731; (b) Simokaitiene J, Grigalevicius S, Grazulevicius J V, Rutkaite R, Kazlauskas K, Jursenas S, Jankauskas V and Sidaravicius J 2006 Synthesis, photophysical and photoelectrical properties of glass-forming phenothiazinyl-and carbazolylsubstituted ethylenes J. Optoelectron Adv. Mater. 8 876; (c) Jin Y, Zhang A, Huang Y and Zhang W 2010 Shape-persistent arylenevinylene macrocycles (AVMs) prepared via acyclic diene metathesis macrocyclization (ADMAC) Chem. Commun. 468258

26. Chen C H, Lin J T and Yeh M C P 2006 Stilbene like carbazole dimer-based electroluminescent materials Tetrahedron 628564

27. Jiao C-X, Shen Q, Huan S-Y, Shen G-L and Yu R-Q 2005 Conjugated carbazole dimer as fluorescence carrier for preparation of iodine-sensitive chemical sensor Anal. Chim. Acta 528229

28. Murali K, Sparkes H A, Pandiyan B V and Rajendra Prasad K J 2017 Synthesis, photophysical properties and DFT analysis of highly substituted pyrido carbazolebased "push pull" chromophores New J. Chem. 418242

29. Maharani S and Ranjith Kumar R 2015 Domino fourcomponent synthesis of novel cycloocta[b]pyridines Tetrahedraon Lett. $\mathbf{5 6} 179$ 